\title{
EchoGéo
}

$34 \mid 2015$

Varia

\section{Critiques et enjeux du Dark tourism à travers la focale sud-africaine}

Fabrice Folio

\section{OpenEdition}

1 Journals

Édition électronique

URL : https://journals.openedition.org/echogeo/14371

DOI : 10.4000/echogeo.14371

ISSN : 1963-1197

Éditeur

Pôle de recherche pour l'organisation et la diffusion de l'information géographique (CNRS UMR 8586)

Référence électronique

Fabrice Folio, «Critiques et enjeux du Dark tourism à travers la focale sud-africaine », EchoGéo [En ligne], 34 | 2015, mis en ligne le 15 décembre 2015, consulté le 31 juillet 2021. URL : http:// journals.openedition.org/echogeo/14371; DOI : https://doi.org/10.4000/echogeo.14371

Ce document a été généré automatiquement le 31 juillet 2021.

EchoGéo est mis à disposition selon les termes de la licence Creative Commons Attribution - Pas d'Utilisation Commerciale - Pas de Modification 4.0 International (CC BY-NC-ND) 


\title{
Critiques et enjeux du Dark tourism à travers la focale sud-africaine
}

\author{
Fabrice Folio
}

\section{Introduction}

1 Le champ de recherche associé à la visite d'endroits liés à la précarité, aux épisodes dramatiques et à la mort est relativement nouveau dans la sphère francophone. En pleine expansion sur la planète, il est davantage étudié dans le milieu anglophone. Entre autres, des auteurs tels que Lennon et Foley (1996) l'ont analysé et théorisé en qualité de nouvelles niches de l'industrie touristique, proposant l'appellation de Dark tourism («tourisme sombre ou noir»). Toutefois, dès lors que l'authenticité de ces prestations serait mise à mal et qu'une «folklorisation" s'instaurerait, il est une littérature qui souligne que le secteur du tourisme peut devenir un vecteur inapproprié et même immoral de la restitution des malheurs, des troubles de l'histoire et de la souffrance humaine (Hewison, 1987 ; MacCannell, 1992).

2 Si cet axe est amené à faire face à des jugements critiques, dans le droit-fil de Poon (1993) et des travaux de Knafou (2012), de Urbain (2011) et de Dewailly (2006), nous considérons que le touriste moderne est, en ce qui le concerne, en quête d'expériences renouvelées et diversifiées qui allient l'émotion, les sensations et l'envie de connaissances, y compris partielles (outre d'autres fondamentaux que sont le ludisme et la sécurité). Il est aussi en demande de rareté et d'exceptionnel et demeure, plus qu'on ne le croit, sensible aux enjeux planétaires (qu'ils soient environnementaux ou civilisationnels). À travers le tourisme, il se construit lui-même et travaille tout un champ d'interrogations personnelles.

En parallèle, l'intérêt des destinations est aujourd'hui d'exhumer des moments forts de leur passé (processus de «patrimonialisation » en vogue) dans un esprit concurrentiel. Cela consiste à les inscrire dans une narration ou un grand récit, source à la fois d'intérêts socio-économiques - le développement économique par le tourisme - et d'enjeux identitaires et politiques - l'affirmation de la fierté et de l'estime par rapport à 
un passé assumé -. Il en va de leur singularisation et de leur positionnement dans l'arène touristique mondiale.

Dans cet article, nous nous proposons d'aborder ce domaine de recherche, en l'illustrant par quelques retours d'expérience du terrain sud-africain. Certaines prestations de la métropole de Johannesburg, située dans la province du Gauteng, seront ainsi examinées. Comme postulat initial, nous soutenons que ce type d'activité s'intéresse à des moments forts et anciennement occultés de l'histoire nationale. Dans le cas sud-africain, nous nous pencherons sur la lutte anti-apartheid. Ce faisant, ces activités tendent à valoriser des lieux qui furent, que ce soit dans un passé proche ou lointain, refoulés et à la réputation marquée, c'est à dire des espaces que nous qualifierons de confins - ou de marges - touristiques. En effet, ceux-ci sont tantôt - ou tout à la fois - localisés en position périphérique et difficilement accessibles (critère localisant), tantôt « appréhendés " pour cause d'image dégradée (critère de marquage).

Derrière ces lieux devenus attractions, une double question tend à affleurer : quelles sont les raisons qui peuvent pousser à leur valorisation et scénarisation? Et le mariage entre la peine, la souffrance et l'industrie du tourisme et des loisirs est-il " concevable»? Nous soutenons que l'extrême pluralité des enjeux sous-jacents doit empêcher tout débat tronqué et lesté d'idées reçues. Des ressorts multiples président à la volonté d'accéder aux prestations du Dark tourism. Nous articulerons notre propos autour d'une dialectique axée d'abord sur l'offre puis sur la demande et ce en alternant petite et grande échelle ${ }^{1}$.

\section{Pour un état des lieux de l'offre du Dark tourism}

\section{Le tourisme sombre : éclectisme des expériences et de la terminologie}

6 Notre objectif est tout d'abord d'offrir un regard général sur ce champ de recherche et sur les enjeux qu'il soulève pour les territoires. Nous proposerons pour ce faire un diagnostic mondial qui reste on en convient fragmentaire, mais passe toutefois en revue des exemples notoires. Nous l'accompagnerons d'un essai de synthèse terminologique.

7 En préambule, nous pouvons citer deux anecdotes. En 2006 une photographie prise au Proche Orient devient célèbre. Pas uniquement pour sa prétendue qualité esthétique en recevant le prestigieux prix World Press Photo-, mais pour les réactions finalement très mitigées qu'elle suscite au sein même du jury (comme dans la presse qui la relaye les jours suivants) : cette berline décapotable remplie de jeunes curieux dans le vent, traversant les décombres de la banlieue de Beyrouth au lendemain du cessez-le-feu négocié entre Israël et le Hezbollah libanais, ne laisse personne indifférent. Autre lieu, deux années auparavant, à Telwatta au Sri Lanka. À la suite du tsunami frappant les rives de l'océan Indien, le déraillement d'un train cause la mort de 1700 personnes. Le site de la catastrophe va intriguer et faire peu à peu sensation par le nombre croissant de visiteurs qui y accourront pour examiner les lieux... et (s'y faire) photographier.

8 Il est des prestations formelles aujourd'hui établies. En Pologne, le site d'AuschwitzBirkenau, de funeste notoriété, s'affiche comme l'une des premières destinations du pays. En 2012, 1,43 million de personnes ont visité le camp (soit 130000 de plus qu'en 
2010). De même, la célèbre Security Prison 21 (S-21) des Khmers Rouges est maintenant accessible au Cambodge via le Tuol Sleng Genocide Memorial. À Elm Street, à Dallas, Texas, un million de visiteurs viennent chaque année photographier ou filmer la rue où John F. Kennedy fut assassiné, en essayant de revivre la scène du 22 novembre 1963. Au Japon, le Hiroshima Peace Memorial Park est un mémorial rendant hommage aux 140000 victimes de l'attaque nucléaire. Là où s'élevaient les tours du World Trade Center à New York, Ground Zero est devenu une plaque tournante du tourisme local, accueillant 3,5 millions de visiteurs chaque année (Kang, Scott, Lee \& Ballantyne, 2012). Que dire en outre des tours opérateurs - Historic New Orleans Tours, Louisiana Tour Co., Tours BaYou...proposant la visite guidée des quartiers détruits de la Nouvelle Orléans par l'ouragan Katrina (Hernandez, 2008) ? Dans la province du Sichuan, le gouvernement chinois propose, pour sa part, des circuits pénétrant au sein même des ruines causées par le séisme de 2008.

Ces scènes d'observation que d'aucuns pourraient trouver incongrues voire déplacées se situeraient-elles à mille lieux de ce que l'on considère généralement comme relevant de la matière touristique ? Les mythiques 35 (Sea Sun Sand) laisseraient-ils la place à de nouveaux 3S, relatifs cette fois au sang, à la sueur... et à la souffrance? Quoi qu'il en soit, elles ne sont plus des cas isolés (si elles ne l'ont jamais été...), comme l'énumération d'exemples évoqués dans cet article tentera de le démontrer. On pourrait dès lors les caractériser comme la matérialisation d'un genre de tourisme de mémoire associé à des drames de l'histoire de l'humanité, ou encore d'une forme de "tourisme de catastrophe ", si tant est que cet oxymoron puisse déontologiquement être accepté académiquement.

Si ses réalités sont multiples, le Dark tourism fait l'objet de courants d'études plutôt récents et de plus en plus féconds, particulièrement dans la sphère anglophone. Ce tourisme qu'on qualifiera donc pour le moment de tragique et/ou de morbide est notamment étudié par le Docteur Philip Stone, professeur en "thanatologie " à l'University of Central Lancashire (UCLan), lui-même directeur de l'Institute for Dark Tourism Research. La conceptualisation du " death, disaster and the seemingly macabre » $\mathrm{y}$ est une réalité. Ce secteur touristique particulier et que l'on connaîtrait moins serait devenu, à le croire, un véritable phénomène de société. Les professionnels du secteur ne s'y trompent d'ailleurs pas : le célèbre guide Lonely Planet présente depuis 2007 un numéro spécial portant sur le Dark tourism. Les prestataires touristiques traditionnels ont eux-mêmes flairé ce filon, en occupant dorénavant le marché dans le cadre d'une diversification tous azimut de leurs produits touristiques. D'autres sont simplement apparus, le plus connu restant sans conteste l'agence britannique Disaster Tourism; cette dernière propose depuis 2010 des « séjours catastrophe » sur mesure, avec à la clé un site internet détaillé sur le sujet. Enfin des sites personnalisés tels que www.dark.tourism.com, www.grief-tourism.com et www.thecabinet.com existent par ailleurs sur la toile.

11 Des instituts de recherche et des chercheurs mobilisés, une industrie du tourisme qui en parle désormais sans tabou... Il existe par conséquent un intérêt à la fois scientifique et économique pour la question. Peut-être parce que s'entremêlent à ce niveau plusieurs enjeux (d'ordre sociologique notamment, nous aurons l'occasion d'y revenir), ainsi qu'une grande part de curiosité, de doutes et finalement d'incertitude sur ses fondements mêmes. Effectivement des dogmes attachés aux motivations et aux pratiques touristiques dites « classiques », traditionnellement associées à la recherche 
de plaisir, à l'allégresse, au relâchement des contraintes et à la légèreté, semblent à tout le moins ébranlés...

12 Ce domaine de recherche a été conceptualisé par plusieurs auteurs. Parmi les plus notoires, il faut citer J. Lennon et M. Foley qui en 1996 popularisent ce terme de «Dark tourism » (soit le tourisme sombre ou noir selon la traduction qui en sera faite dans la littérature francophone). Ils le définissent comme un "phénomène qui embrasse la mise à disposition et la consommation de sites authentiques marqués par la mort et les désastres ». Cependant, d'autres expressions existent. Les plus marquants incluent le «thanatourisme ${ }^{2}$ » (Dann \& Seaton, 2003), le «tourisme morbide» (Blom, 2000), le " tourisme de points noirs" (blackspot tourism, Rojek, 1993) ou encore le "tourisme de tristesse » (O'Neill, 2002). D'après Sharpley et Stone (2009), les sites du tourisme sombre sont relatifs «à la mort, à la souffrance et au macabre ». Si l'on reprend la définition de Marcel (2003, in Novelli, 2005), cette activité s'apparente à une forme de «visite d'endroits où des tragédies voire des décès soulevant une vive émotion se sont déroulés, lesquels continuent d'avoir une influence sur nos vies ». Pour Macdonald (2009), les lieux concernés sont liés à l'étrangeté, à l'incongruité et à la controverse. L'auteur insiste sur le fait qu'ils devraient être classifiés comme «sites patrimoniaux difficiles ». Enfin pour White et Frew (2013), la visite des ghettos et bidonvilles de la planète - dans les pays développés comme dans ceux en développement - peuvent aussi relever d'une forme d'attractions touristiques dites sombres. Un timide consensus se dégage donc sur le fait que le Dark tourism se réfère à une forme de visites associées à l'affliction, aux tragédies et à la détresse sociale et économique, en dépit du fait que la nature humaine a généralement tendance à fuir tous drames et malheurs.

\section{Une mise en tourisme historico-sensationnelle « mainstream » : essai de typologie}

13 Ce type de tourisme fait florès depuis la fin des années 1990 et le début des années 2000 et correspond à l'intérêt manifesté à cette période pour les sites de catastrophes subites (naturelles ou anthropiques). Pourtant, l'approche de sites historiques liés à la mort existait auparavant ! En effet, les monuments commémoratifs de guerre, les prisons, les cimetières, les champs de bataille et les forteresses ou encore l'héritage esclavagiste, tous ayant un lien avec la mort (mais aussi la souffrance et la peine), sont pour certains pris d'assaut depuis fort longtemps. S'il fallait tenter de brosser une esquisse typologique des prestations concernées, des choix doivent s'opérer et les critères à retenir deviennent forcément subjectifs.

Dark tourism n'est pas spécifique à une partie du monde. Il opère toutefois et c'est assez logique là où le secteur touristique est le mieux implanté. En revanche, la temporalité semble jouer, les actes plus récents étant davantage représentés et (re)mis en scène. En outre, les lieux concernés mais aussi les prestations demeurent très disparates. Par conséquent, un travail académique de structuration devient nécessaire.

De prime abord, les endroits et monuments intéressés sont eux-mêmes hétéroclites : cela peut en effet être une stèle ou un cénotaphe, une maison ou un bâtiment, un musée, une rue, une zone rurale, voire une ville ou un littoral (mais aussi un pays tout entier !). À tout le moins, peut-on déjà proposer une distinction entre d'un côté des espaces ouverts (comme un champ de bataille...) et de l'autre des endroits fermés (le cas d'un centre d'interprétation...). 
16 Selon Dunkley (2005), on peut distinguer un panel de 7 catégories se déclinant sous un "parapluie thanatouristique ». L'auteur croise lieux/types d'évènement/sentiments ressentis :

- le fright tourism (frisson) : par exemple l'univers autour de Vlad Dracula...

- le grief tourism (peine) : le cimetière d'Arlington ou du Père Lachaise

- le hardship tourism (épreuve) : la prison de Robben island ou d'Alcatraz

- le tragedy tourism (drame) : Hiroshima ou les Tsunami tours de Banda Aceh

- le warfare tourism (guerre) : les plages du D-Day et autres champs de bataille

- le genocide tourism : le cas du Rwanda

- le «thanatourisme extrême»: les exécutions publiques et inhumations célestes qui se déroulent en Inde ou au Tibet.

17 En poursuivant notre réflexion, on peut sans doute retenir quelques couples assez simples, destinés à resserrer et mieux classifier ce spectre de possibilités. Nous proposons un panel d'axes thématiques organisés autour des grandes caractéristiques du lieu concerné :

- l'ancien/le contemporain

- le naturel/l'anthropique

- l'inoccupé/l'habité

- le site conçu à cette fin/qui l'est devenu accidentellement

- le lieu davantage centré sur l'histoire/la mémoire ou sur la mort/la souffrance (bien que la frontière entre les deux soit parfois fine comme on le verra plus loin).

Ceci établi, on peut à ce moment tenter de structurer des sous-branches, qui seraient alors une amorce de typologie du Dark tourism (tableau 1).

Tableau 1- typologie du Dark tourism

\begin{tabular}{|l|l|}
\hline \begin{tabular}{l} 
Forme $\begin{array}{l}\text { Dark } \\
\text { tourism }\end{array}$ \\
\hline 1
\end{tabular} & tourisme sombre lié à des batailles historiques majeures \\
\hline 2 & $\begin{array}{l}\text { tourisme sombre associé à des périodes d'oppression, à des régimes sanguinaires et/ } \\
\text { ou à des massacres de masse }\end{array}$ \\
\hline 3 & $\begin{array}{l}\text { tourisme sombre lié à des homicides notoires ou à des accidents funestes touchant } \\
\text { des personnalités }\end{array}$ \\
\hline 4 & $\begin{array}{l}\text { tourisme sombre qui concerne des catastrophes naturelles et/ou humaines plus ou } \\
\text { moins récentes }\end{array}$ \\
\hline 5 & $\begin{array}{l}\text { tourisme sombre se déroulant au sein de secteurs contemporains vus comme } \\
\text { paupérisés/dangereux/instables de la planète (bidonvilles, ghettos... mais aussi des } \\
\text { destinations comme Mexico, San Salvador ou la Corée du Nord !). }\end{array}$ \\
\hline
\end{tabular}

Nous excluons ici toutes formes basées prioritairement sur la fiction et sur l'amusement, pour ne retenir que la retranscription des faits authentiques (ou du moins présentés de la sorte). Nous intégrons par ailleurs le tourisme pro-pauvre (Rogerson, 2006), se déroulant au sein d'espaces vécus actuels. 
19 À noter que A. Kendle (2008) a pu rajouter deux sous-secteurs plus discutables: le Doomsday tourism concernerait des sites en voie de disparition pour cause de dérèglement climatique (comme le Kilimandjaro) et de la remontée du niveau marin (certaines îles des Maldives). Bien plus controversée est sa catégorisation du Suicide tourism. Ce dernier concernerait les services liés à l'euthanasie (accessibles dans certains pays comme la Suisse) et seraient aussi rangés dans cette sous-catégorie les déplacements volontaires dans des lieux lointains, afin de s'y donner la mort (par exemple un pont connu...). Certains auteurs (Sharpley, 2005 ; Stone, 2006) ont, eux, établi des degrés intéressants de palettes de couleur (Dark, Darker, Darkest ou encore Pale, Grey, Black...) au vu des prestations offertes, en distinguant notamment l'intensité dramaturgique, mais aussi dans le cas de Sharpley la nature - authentique/non authentique et donc éducative ou ludique - de l'expérience.

Indéniablement la notoriété de ces sites est assurée par la combinaison de deux éléments : en premier lieu leur impact historique à grande et à petite échelle (et plus celui-ci est récent plus il a l'opportunité d'être valorisé) ; en second lieu l'éclairage médiatique et académique qui les a fait connaître et les a ensuite popularisé. Ce second facteur joue à plein dans un contexte de mondialisation et ce depuis plus d'un quart de siècle. Ces sites fascinent d'autant plus qu'ils ont acquis une réelle célébrité. Pour le sociologue britannique J. Lennon, directeur du Moffat Centre for Travel and Tourism Business Development à Glasgow, les médias et les productions cinématographiques ont notamment une responsabilité majeure dans l'attirance pour le macabre. Nous pouvons citer deux exemples: le nombre de visites enregistrées au camp d'Auschwitz aurait augmenté de façon notable avec la sortie en salle du film de Steven Spielberg «La Liste de Schindler». Il en est de même pour le bidonville de Dharavi à Mumbaï. Son « désenclavement » fait suite au long métrage multi-oscarisé « Slumdog millionaire ».

Ces pratiques s'appuient sur un savant mélange de ce qui est rare, nouveau, exceptionnel et de l'avènement de ce que l'on pourrait considérer comme une envie de culture et d'histoire sensationnelle, mais en même temps populaire et relativement dépouillée (ou du moins allégée de certaines nuances). Nous proposons à cette fin l'expression « histoire maintream ». Cette même histoire que l'on cherchera par la suite à confronter à son image mentale, c'est à dire façonnée par tout ce que l'on a pu apprendre, lire, scruter sur le sujet.

Le regard sur le phénomène ne laisse en tout cas pas indifférent. Des lieux de mémoire touristiques difficiles, en particulier ceux consacrés à des génocides (de Kigali à Auschwitz), peuvent être considérés comme indécents. Ils provoqueraient un certain malaise face à un héritage pour lequel il conviendrait de demeurer pudique. Ces lieux de mémoire sont devenus des produits d'appel pour des tours opérateurs parfois peu scrupuleux. Il est vrai que ces prestations ont occasionnellement été rajoutées comme outil marketing supplémentaire sans guère, semble-t-il, de considérations déontologiques. Au pays des mille collines par exemple, les onéreux "Gorillas Tours " s'associent de façon décomplexée à un "Tour du génocide rwandais " articulé sur le Murambi Genocide Memorial Centre. Auschwitz, quant à lui, est un puissant produit d'appel qui capitalise sur son nom, jouant sans vergogne de ce mélange d'attraction et de répulsion. Dès l'aéroport de Cracovie, des opérateurs locaux offrent l'aller-retour en 3 heures pour une vingtaine d'euros! Le camp côtoie de la sorte la mine de sel Wieliczka ou la Cracovie du XVII e siècle à l'intérieur des brochures touristiques... 


\section{La focale sud-africaine : plusieurs fers de lance et un agenda politique}

En Afrique du Sud ${ }^{3}$, le tourisme dit de mémoire a le vent en poupe. La valorisation de l'histoire mouvementée du pays est présente dans les catalogues touristiques. Elle est appuyée par une politique étatique qui joue explicitement de la carte du tourisme patrimonial dans une visée de reconstruction nationale et de développement local par le tourisme. Les prestations concernées correspondent bien au Dark tourism, même si le terme lui-même reste peu usité. Notons que ce tourisme mémoriel difficile ne s'intéresse pas qu'à la période de l'apartheid, mais aussi aux périodes antérieures (guerres des Boers, guerres de la conquête coloniale...). Toutefois les lieux liés à la politique de développement séparé sont très représentés (tableau 2). Le pays affronte cette page d'histoire difficile et témoigne de ce moment via plusieurs monuments : mémorial, stèles, quartiers, prisons (Ashworth, 2004).

Tableau 2 - Essai de classification des espaces du Dark tourism en Afrique du Sud

\begin{tabular}{|l|l|}
\hline $\begin{array}{l}\text { Les musées culturels et } \\
\text { historiques et notamment } \\
\text { ceux d'apartheid (Swarns, } \\
\text { 2001) }\end{array}$ & $\begin{array}{l}\text { Parmi les plus fréquentés citons l'Apartheid Museum à Johannesburg, } \\
\text { le Hector Pieterson museum à Soweto, le District 6 et le musée Bo Kaap à } \\
\text { CapeTown, le Red Location à Port Elisabeth ou encore le KwaMuhle } \\
\text { museum à Durban/eTheKwini'. }\end{array}$ \\
\hline $\begin{array}{l}\text { Les sites de champs de } \\
\text { bataille }\end{array}$ & $\begin{array}{l}\text { Ils sont pour l'essentiel liés à l'histoire coloniale entre Anglais, Boers, } \\
\text { Xhosas, Zulu etc. Les battlefields les plus illustres sont Isandhlwana et } \\
\text { Rorke's Drift (tout deux liés aux guerres anglo-zouloues), mais aussi } \\
\text { Blood river où s'est déroulée une bataille épique entre Boer et Zoulous } \\
\text { dans la province du KwaZulu-Natal (Seaton \& Lennon, 2004). }\end{array}$ \\
\hline $\begin{array}{l}\text { Lesisons } \\
\text { "politiques " dites }\end{array}$ & $\begin{array}{l}\text { Au premier rang desquelles on trouve l'île de Robben island au large } \\
\text { du Cap (où Nelson Mandela a passé 18 de ses 27 années de captivité } \\
\text { en compagnie d'autres activistes anti-apartheid comme Walter } \\
\text { Sisulu), ainsi que le Constitution Hill non loin du quartier péricentral } \\
\text { de Hillbrow à Johannesburg (des personnalités y ont été incarcérées } \\
\text { telles que Gandhi, Bram Fischer ou Winnie Mandela, parfois dans } \\
\text { l'attente d'un procès comme ce fut le cas pour Nelson Mandela lors } \\
\text { du Rivonia trial de 1963). }\end{array}$ \\
\hline $\begin{array}{l}\text { Township tours, émarge à } \\
\text { cette catégorisation selon la } \\
\text { terminologie élargie de } \\
\text { certains auteurs }\end{array}$ & $\begin{array}{l}\text { Dans le pays, ceux de Johannesburg (Alexandra et surtout Soweto), } \\
\text { du Cap (Langa, Gugulethu) et à degré moindre de Durban (Inanda, } \\
\text { Umlazi) sont les plus arpentés. }\end{array}$ \\
\hline
\end{tabular}

Quantité de prestataires privés s'approprient le secteur et certains souscrivent à la politique du Back empowerment (aides et encouragements à l'entreprenariat noir). Les clientèles principalement visées sont d'une part un public étranger ultramarin ; d'autre part un public local spécifique lié au monde éducatif, associatif ou professionnel. L'ensemble s'articule donc autour des sorties scolaires, du tourisme d'affaires et de 
l'agrément. L'originalité de la focale sud-africaine se comprend de la manière suivante : l'économique et le politique sont intimement liés pour l'écriture - ou la réécriture d'un grand récit national porteur d'espoir. Elle participe pleinement à la fabrication de la mémoire sud-africaine (Nuttall, Coetzee, 2000). Pour le démontrer, attardons-nous sur trois exemples notoires de la capitale économique de Johannesburg, métropole de 4,4 millions d'habitants.

\section{Le musée de l'apartheid ou le teach by showing}

25 À la suite de la transition démocratique, l'État sud-africain, avec à sa tête l'ex-parti de la libération l'African National Congress (ANC), a décidé d'investir les musées sudafricains d'une noble mission: véhiculer connaissance mais aussi fierté de l'histoire nationale. Les musées de la nation arc-en-ciel entendaient ainsi agir en tant que lieux symboliques d'une nouvelle narration d'obédience réparatrice et optimiste. Nombre de ces musées sont devenus des points de départ à l'exploration des quartiers réservés anciennement à la population noire, les townships (cf. infra). Ils s'apparentent donc à la fois à des institutions culturelles et à des sites touristiques, et ce alors même qu'ils étaient plutôt par le passé des espaces « craints » et répulsifs. Ce furent fréquemment des prisons politiques ou même des "maisons indigènes " pour migrants urbains, comme dans le cas du KwaMuhle Museum à Durban.

Le musée de l'apartheid se situe dans les faubourgs sud de Johannesburg. Il a émergé ex-nihilo dans un contexte transitoire en 2001. Sa vocation était de revenir sur les pages sombres du pays, en endossant les standards des musées de classe mondiale. Son origine est assez cocasse car il est en réalité lié à la naissance d'un vaste parc à thème : celui de Gold Reef City. Désireux de créer un parc d'attractions organisé autour de l'univers des mines d'or dans la périphérie de Johannesburg, ses concepteurs - la compagnie Akani-eGoli - ont dû faire face à l'injonction municipale d'insérer un projet à vocation sociale dans leur initiative. De là provient ce musée pédagogique privé, perçu alors comme le mieux à même d'incarner un contrepoint didactique au monde fantaisiste du parc, tout en créant des emplois et en stimulant l'économie locale. L'origine du musée est donc davantage associée à des motifs économiques, devenus ensuite peu à peu symboliques et politiques (Leibowitz, 2008).

Les architectes se sont inspirés du Musée Mémorial de l'Holocauste à Washington. Devenus les agents de la re-imagination et de la représentation historique (Bremner, 2007), ils ont orienté leur travail - en terme de fil scénaristique et des matériaux utilisés - sur une structure évolutive assez moderne. La place du son et de l'image y est marquée. Le visiteur est plongé dans un environnement austère et oppressant (en particulier dans la première moitié du circuit), rappelant le monde carcéral ou celui des camps. L'objectif initial était donc d'associer projet pédagogique et projet performatif (Fassin, 2007). Il s'agit de retranscrire un environnement âpre au travers d'une expérience sensorielle mêlant la désorientation et la brutalité ; et dans le même temps, d'assumer un côté ludique, matérialisé entre autres par la double entrée réservée aux personnes blanches ou non-blanches (illustration 1a), la route des migrants (Journeys) et sa succession de miroirs, l'entrelacs de corridors grillagés ou encore les hautparleurs crachant des voix d'époque. Vers la fin, les travées espacées et l'ouverture sur un vaste jardin en hauteur - A place of Healing -, témoignent d'un pays qui a tourné la page d'une ère répressive (illustration 1b). L'architecture contribue explicitement et 
passivement à l'image d'une nouvelle nation unifiée (illustration 1c). La stratégie employée est celle du " teach by showing ». Cette dernière assume un positionnement dans l'industrie des loisirs et un discours dense à l'attention d'une audience internationale comme locale.

Pour immersif qu'il soit, le musée n'est toutefois pas sans présenter une version ambigüe de l'Histoire nationale. En effet, il confine une page décisive du passé sudafricain à une narration quelque peu linéaire et simplifiée. L'ex politique de discrimination raciale devient un événement borné, circonscrit à un temps révolu. Or, ses impacts sur le présent sont toujours prégnants pour une majorité d'individus. De sorte que les visiteurs étrangers ressortent plutôt satisfaits - bien qu'épuisés - de la visite du musée (selon les enquêtes de Kirshenblatt-Gimblett, 1998). Mais pour certains Sud-Africains Noirs, le musée de l'apartheid affiche une tonalité mélodramatique et quelque peu déconnectée du monde réel (Findley, 2005). Ce constat vaut surtout pour les expositions permanentes. Il est intéressant de signaler que, parmi les récentes expositions temporaires, figurent des connexions avec les épisodes pré-apartheid et même avec la page précoloniale (à travers l'empire zoulou par exemple). Qui plus est, cette vision suggestive et artistique du passé ne s'émancipe pas d'un regard parfois biaisé, au sens où un certain nombre d'actes ou d'acteurs sont passés sous silence (ou à tout le moins minorés). Peut-on évoquer le souhait de ne pas trop complexifier la prestation? Au demeurant, une facette afrocentriste - les oppresseurs au pouvoir face aux victimes noires - se dégage (Samarbakhsh-Liberge, 2000). On peut aussi remarquer que les membres de l'ANC y sont célébrés, au détriment d'autres groupes de résistance (les rivaux historiques que sont le PAC et l'AZAPO notamment).

Illustration 1a - I'Apartheid Museum à Johannesburg : entrée

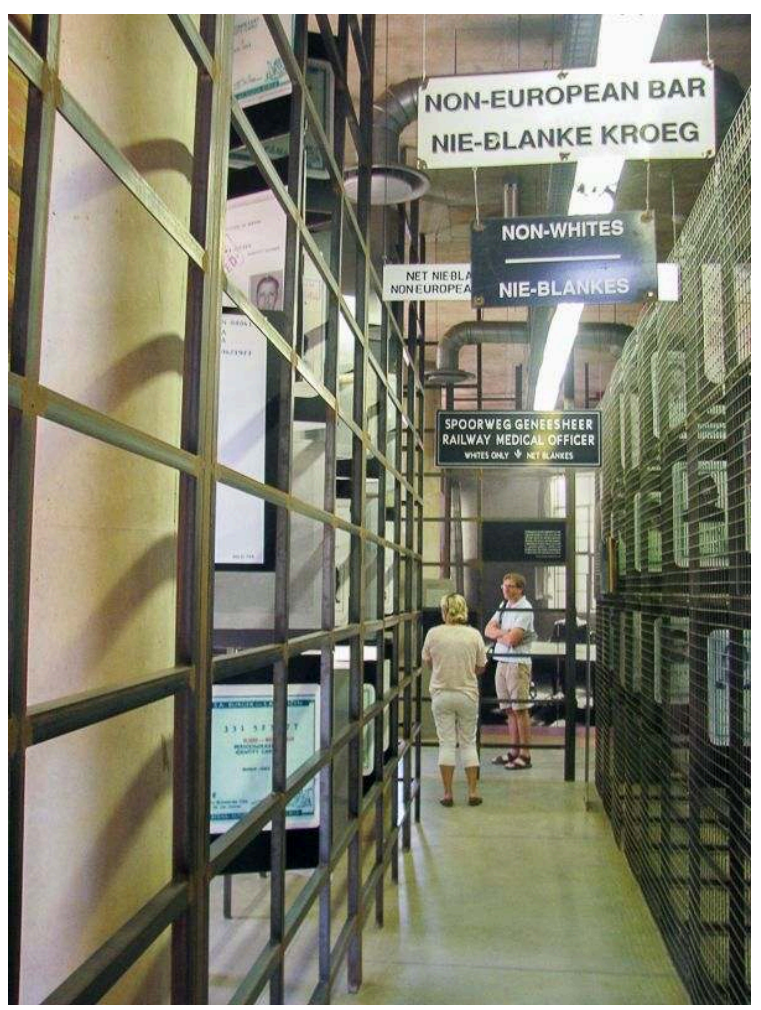

L'atmosphère oppressante de la ségrégation raciale est mise en scène au début du musée. Source : F. Folio. 
Illustration 1b - L'Apartheid Museum à Johannesburg : sortie

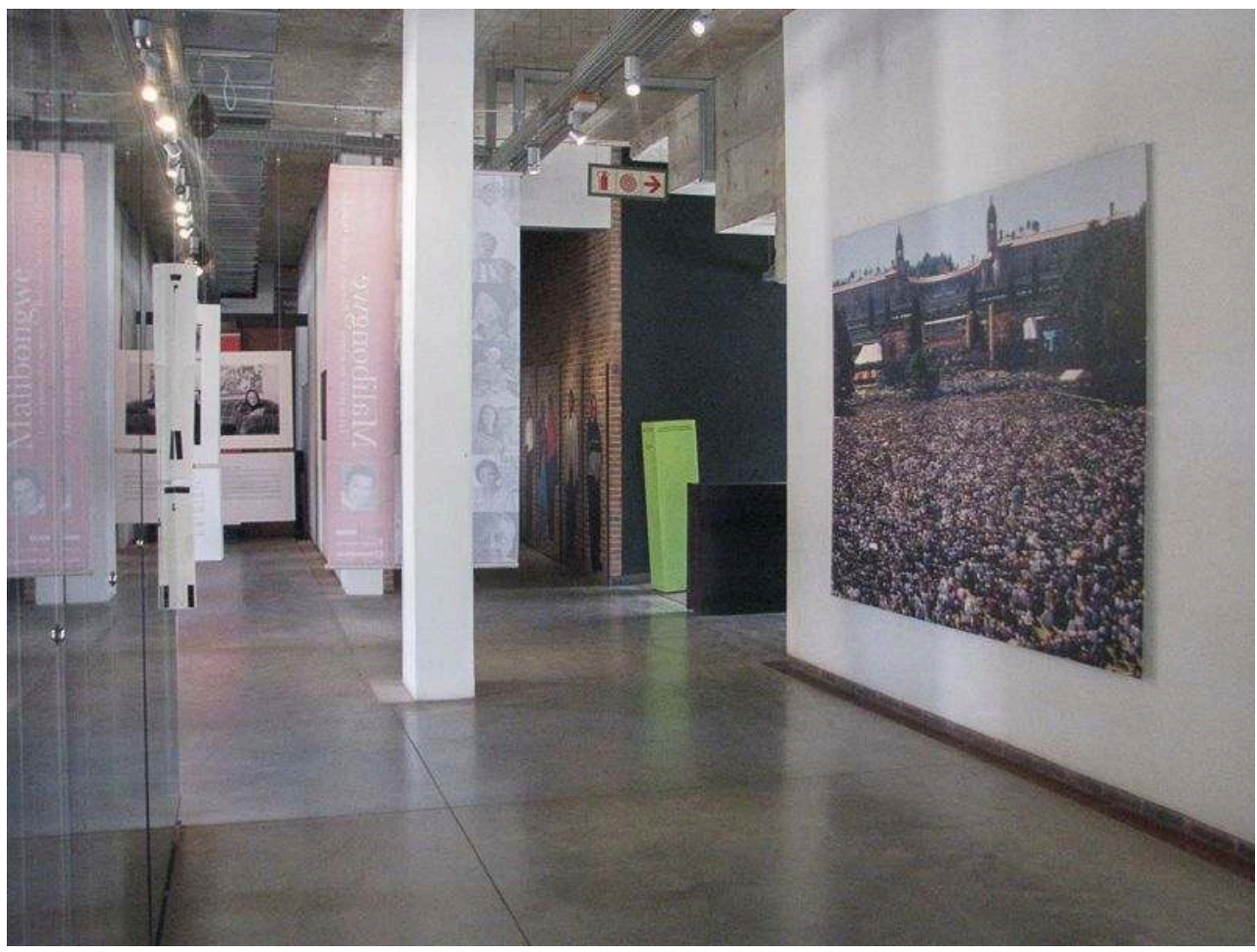

La nouvelle Afrique du Sud postapartheid, ouverte et « éclairée », est dépeinte à la fin du circuit. L'architecture rencontre le symbolique.

Source : F. Folio. 
Illustration 1c - l'Apartheid Museum à Johannesburg : plan

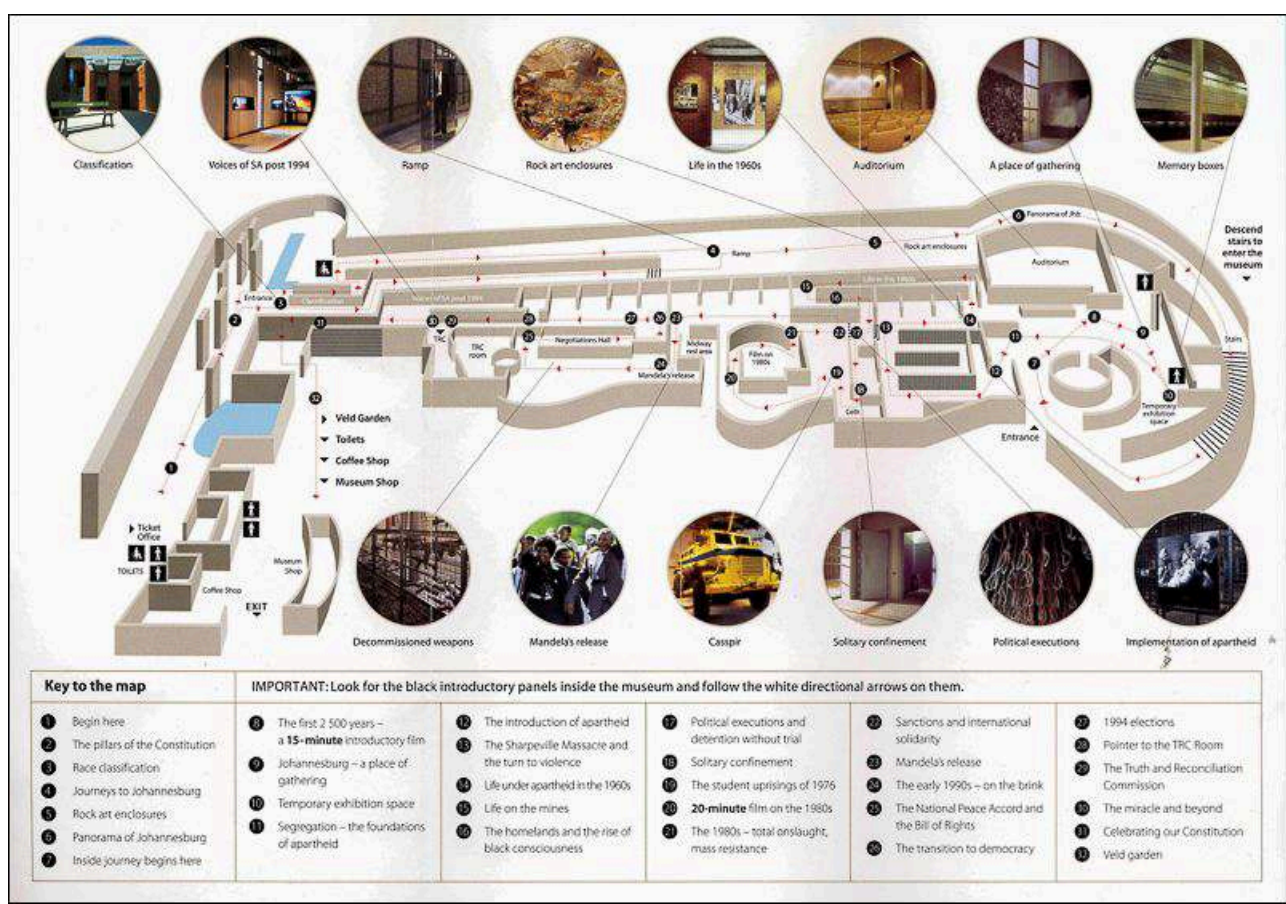

Sur la vue en plan, on discerne l'intérieur du musée et ses éléments constitutifs. Contenus tangibles et intangibles s'entremêlent. L'itinéraire est étiré et labyrinthique. Imposé au visiteur, il suit un ordre chronologique.

Source : brochure d'entrée.

\section{Le Constitution Hill ou quand le(s) passé(s) rencontre(nt) le présent}

Le Constitution Hill incarne une prestation culturelle et historique pour le moins inattendue. Certes, le site ne détient pas la même notoriété touristique que l'Apartheid museum ou Robben island au Cap par exemple. Mais sa singularité tient au fait que ce sont en partie des citoyens ordinaires qui sont amenés à le fréquenter. Le Constitution Hill a été ouvert au public en 1995 dans le cadre d'une structure semi-publique (Constitution Hill Foundation, 2006) : le site fait ainsi fait partie des Blue IQ project de la province du Gauteng, c'est-à-dire des lieux d'investissements stratégiques. Il est également géré par l'organisme public Johannesburg Development Agency (JDA), lequel pilote des initiatives de développement et de réhabilitation urbaine en partenariat avec le secteur privé.

Le Constitution Hill est localisé en zone péricentrale nord de Johannesburg, plus précisément aux abords du quartier - à la réputation pour le moins affirmée - de Hillbrow. Il s'agit d'une zone où sont particulièrement implantées les communautés migrantes, notamment d'Afrique de l'ouest. Le site lui-même se décompose en plusieurs bâtiments : le Old Fort est une ancienne prison de haute sécurité pour les Blancs durant l'apartheid, mais aussi et avant cela le célèbre poste de défense des Afrikaners de $P$. Kruger durant la guerre les opposant aux Anglais (1899-1902). Quant à la Number 4/5, de triste réputation, il incarne la prison indigène : ici s'entassaient comme des « sardines " les populations de couleur. Y ont notamment été retenus des prisonniers et activistes pro-démocrates : outre Robert Sobukwe, on peut citer Gandhi durant sa campagne de résistance. Un peu plus loin, le Women's Jail, représente l'ex-prison réservée aux 
femmes, où ont notamment été incarcérées Winnie Mandela et Albertina Sisulu. Aux côtés de ce patrimoine "dissonant $»^{5}$, l'actuelle cour constitutionnelle a été établie directement sur le site en 2004. L'objectif était à ce moment de constituer un puissant symbole de juxtaposition physique : les abus du passé (prisons) se devant de côtoyer l'espérance en le futur (la cour).

31 De sorte que le Constitution Hill vise à transformer une page d'Histoire chargée négativement en une ode unitaire à l'opiniâtreté et au courage au sein de l'Afrique du Sud postapartheid. On peut aisément énumérer la totalité des effets de niche recherchés (illustrations $2 \mathrm{a}$ à $2 \mathrm{~d}$ ). Ils sont d'ordre (socio-)économique, identitaire et politique :

-Présentée comme une prestation «intégrale», le Constitution Hill touche d'abord à plusieurs périodes (coloniale, apartheid...) et épisodes phares de l'histoire du pays : guerre Anglo-Boers, rassemblement civique des Indiens, lutte - des Noirs mais pas seulement contre la politique de ségrégation raciale.

- Il cible des segments à la fois genrés et communautaires (scénarisation de bâtis pour les Noirs, les Indiens, les Blancs ; pour les femmes, pour les hommes...).

- Il participe en outre à une amorce de régénération urbaine par son établissement aux portes d'un quartier dégradé, à la forte économie souterraine (Hillbrow). Même si les résultats de cette politique méritent encore d'être vérifiés (de forts contrastes paysagers demeurent apparents), on peut tout du moins attester de l'apparition d'une nouvelle forme d'urbanité et d'un « recodage » de l'espace métropolitain par le tourisme et la culture (Nahrath, Stock, 2012).

- Et surtout, il allie passé et présent avec un site judiciaire toujours actif, qui amène des résidents ordinaires à le fréquenter et ainsi à en savoir davantage sur l'histoire du pays (dans une démarche qui n'est plus, on le convient, touristique).

Les icones de l'histoire sud-africaine et les grandes dates de son passé sont une nouvelle fois pleinement mobilisées. La réappropriation patrimoniale est institutionnelle et légitime la puissance publique locale et régionale (Guinard, 2011). Le Constitution Hill amène qui plus est à rester prudent sur la dénonciation de l'union insolite entre évènements tragiques et marchandisation touristique et de loisirs ${ }^{6}$. De manière triviale, on pourrait se demander si un tel site nécessite une boutique de souvenirs ou un restaurant. Mais à partir du moment où ce lieu se démocratise, s'affiche et s'ouvre au grand public, il rentre de facto dans la bulle touristique. Et cette dernière reste un secteur économique : rien d'étonnant donc au fait que les questions philanthropiques ou mémorielles ne soient pas les seules à être prises en considération. Au reste, on pourrait arguer que ce n'est pas la mort stricto sensu rattachée à cet endroit mais bien son histoire pesante et grave qui est commercialisée. Par ailleurs, le site accueille de nombreuses manifestations culturelles et scientifiques dont le déroulé n'est en rien perturbé, d'après nos observations de terrain. Tout d'abord, le complexe est vaste, aéré et compartimenté. Sans doute même trop : le manque de liaison entre chacune de ses composantes et le déficit de cohérence dans sa narration globale sont par exemples critiqués, les différents bâtiments ayant fait l'objet de travaux d'aménagements différents portés par autant d'acteurs (province, municipalité...)! (King, Flynn, 2012). Il $\mathrm{y}$ règne un silence monacal et respectueux. La visite peut s'effectuer seul ou avec un médiateur culturel. Quant aux endroits dits de " repos » (restaurant, librairie mais aussi banc et jardin), ils apparaissent plutôt comme des sas de relâchement après la tension 
emmagasinée. Ils semblent plutôt appréciés des visiteurs, qui peuvent là se poser et discuter plus aisément de leur ressenti.

Illustration 2a - Constitution Hill : plan

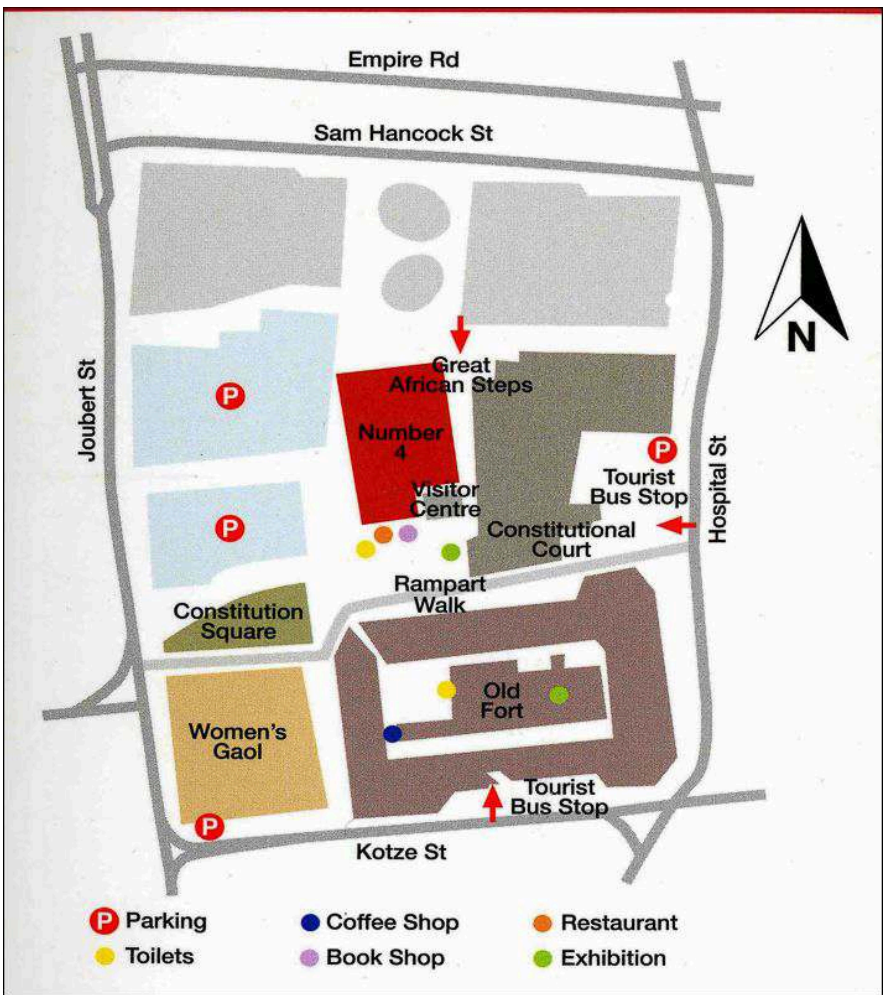

Délimité par quatre rues, le site est très étalé et compartimenté ; à l'héritage patrimonial, se sont ajoutés des équipements culturels et touristiques.

Source : brochure d'entrée. 
Illustration $2 \mathrm{~b}-$ Constitution Hill : sordides cachots de la prison Number 4

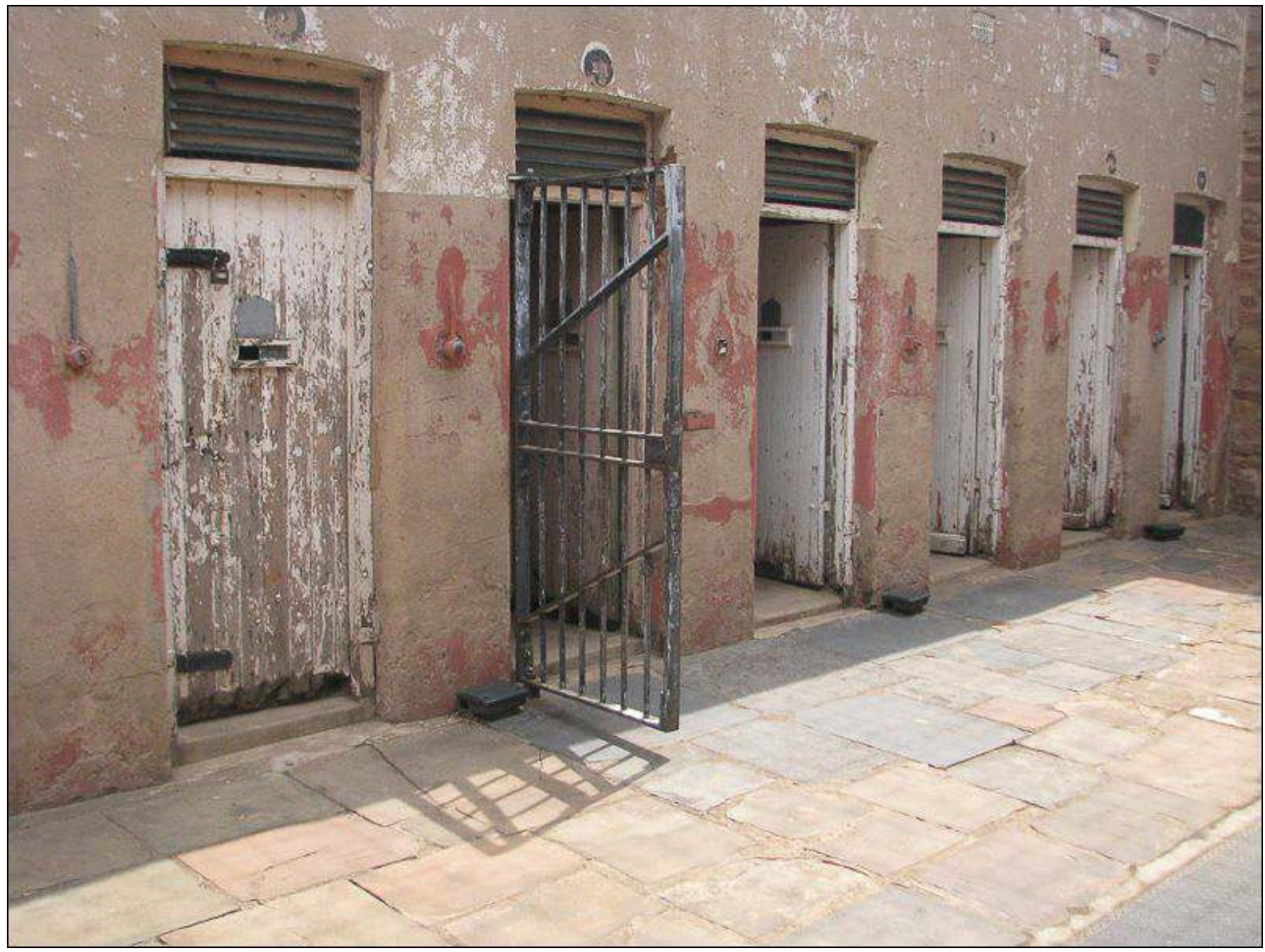

Source : F. Folio, 2014

Illustration 2c - Constitution Hill : mise en scène de la vie du Prisoner of Conscience : le Mahatma Gandhi

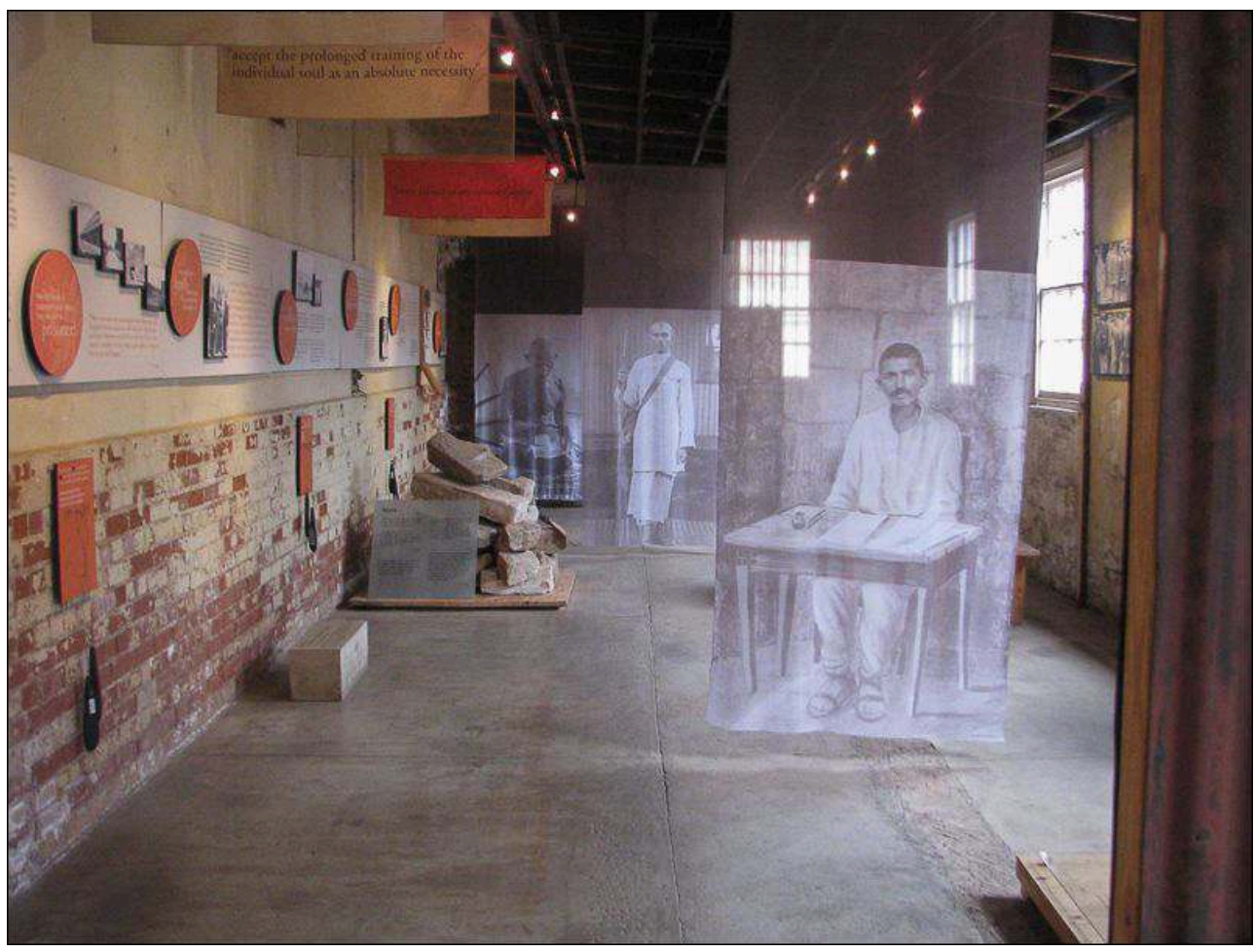

Source : F. Folio, 2014. 
Illustration $2 \mathrm{~d}$ - Constitution Hill : salle d'accueil de l'actuelle cour constitutionnelle sud-africaine

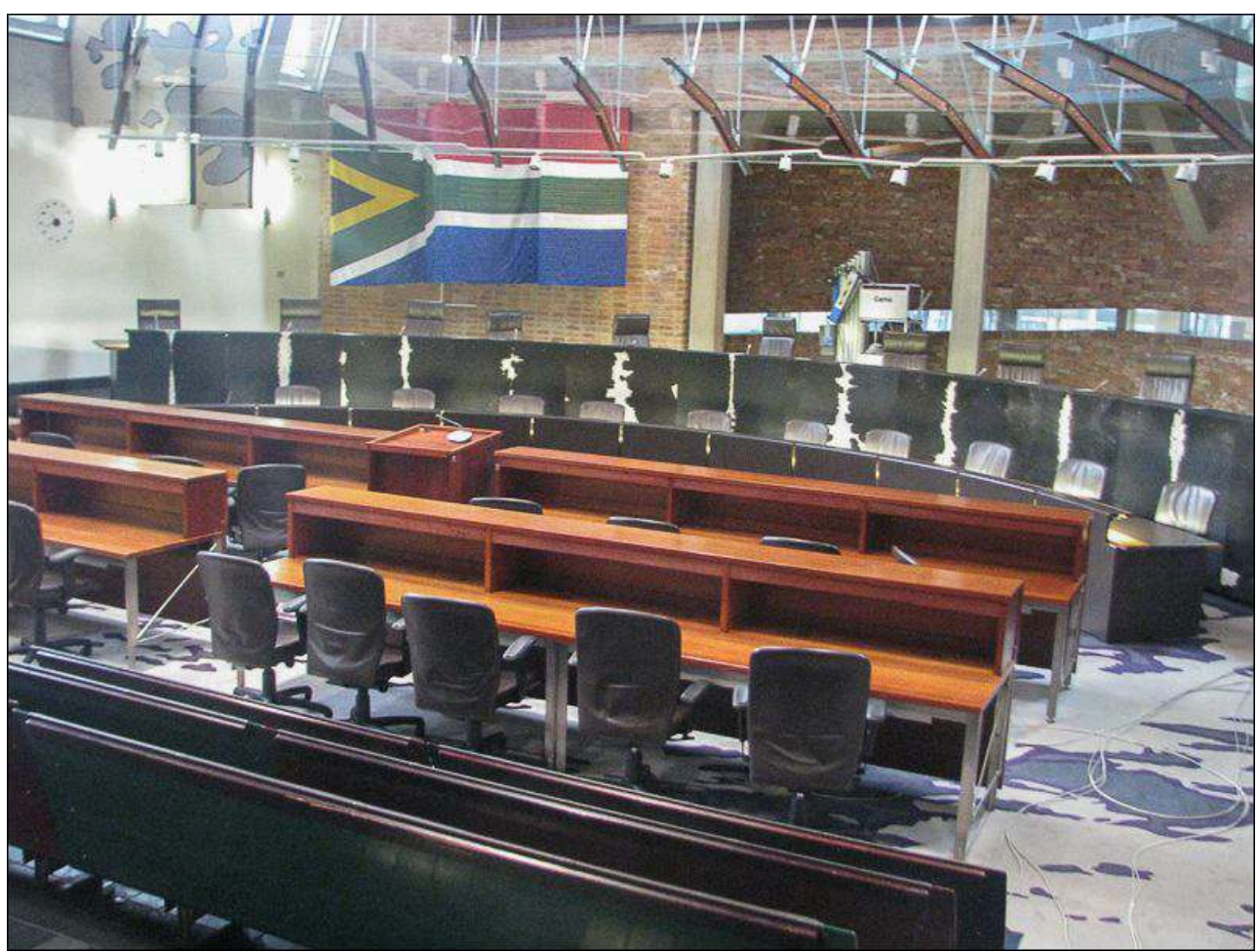

Source : F. Folio, 2014

\section{Les Soweto Tours : la fixation de «bornes » dans un environnement urbain bouillonnant}

Les Township tours sont des attractions touristiques à présent bien installées dans le paysage urbain de l'Afrique du Sud. Il s'agit d'une niche touristique apparue dans les années 1980 à Johannesburg et qui a connu un vif essor ces dernières décennies. Le tout prend place au sein d'un secteur en mutation et confronté à une clientèle diversifiée, curieuse et exigeante. Dans un contexte de mondialisation démultipliant les flux touristiques et aggravant tout à la fois les inégalités de richesse, des sous-espaces mondiaux incarnant le "Sud » s'ouvrent à des visiteurs du «Nord», dans un esprit singulier de curiosité et de désir de compréhension. Rappelons que ces «tours " se déroulent actuellement tant dans les bidonvilles indiens que dans les favelas brésiliens ou autres ghettos nord-américains. Localement, les principaux contingents de touristes sont là encore plutôt étrangers ultramarins (Folio, 2010). Les Township tours sont également associés à l'évènementiel dans le cadre du tourisme d'affaires se déployant depuis le pôle économique de Sandton.

Soweto concentrent des étapes incontournables du circuit. Celui-ci s'étale sur une demi-journée et reste essentiellement motorisé, entrecoupé de quelques haltes. Il est mené par des compagnies privées agrées qui véhiculent les touristes en taxi-minibus confortables. Le guide est souvent un habitant originaire du township ou en tout cas qui le connaît bien. Quant aux lieux spécifiques abordés durant le tour, on trouve tout d'abord Vilakazi street dans le secteur d'Orlando West. Il s'agit de l'artère de la résistance estudiantine où la célèbre manifestation du 16 juin 1976 (contre l'apprentissage obligatoire de l'afrikaans) s'est tenue. Cette étape est associée à un arrêt au Mémorial et 
au musée Hector Pieterson (respectivement ouvert en 2001 et 2002) qui traitent de l'histoire de l'apartheid (illustration 3a). Toutefois, la rue est également "vendue » comme celle où résident - ou ont résidé - deux prix Nobel de la paix (seule rue au monde de ce genre) : les maisons de Nelson Mandela et de Desmond Tutu s'y trouvent en effet (illustration 3b). Plus loin, le tour va immanquablement s'arrêter au Walter Sisulu Square, où a été signée la Freedom charter en 1955. Le square été récemment réaménagé par la municipalité avec la construction d'une vaste esplanade et d'un hôtel haut de gamme (illustration 3c). À proximité, par-delà la voie ferrée, les bidonvilles se déploient comme pour mieux rappeler la réalité éminemment disparate et inégale de l'Afrique du Sud postapartheid (illustration $3 \mathrm{~d}$ ). Le reste de Soweto n'est visible qu'assez fugacement.

Il convient donc de préciser que la totalité du township n'est pas couverte par cette entreprise de touristification. À travers ces «bornes" ou lieux remarquables, le visiteur n'en verra finalement qu'une petite partie, au sein de tours maintenant bien rodés et établis, où la population locale s'est habituée à voir se succéder les autobus et véhicules privés et à voir déambuler les touristes avec leur téléphone intelligent et autre tablette numérique. Ce sont d'ailleurs à ces étapes phares du tour - on peut évoquer l'idée d'une visite en saut de puce -, que restaurants typiques, nouveaux bed and breakfast, sheeben (ex-bars clandestins) et autres commerces ambulants (artisanat et articles de souvenirs) se sont arrimés.

Dans les enquêtes réalisées par T. Mudzanani (2014) au Mémorial Hector Pieterson, l'auteur avance que de multiples paramètres éclairent le désir d'approcher le célèbre quartier de Soweto. Nous y reviendrons plus loin. Mais s'il est vrai que ce dernier est une allégorie du combat anti-apartheid, il se détache grâce à son image de marque. La rue Vilakazi est particulièrement prisée, car elle renvoie, au-delà de la marche des écoliers contre l'enseignement de la langue afrikaans, au fameux cliché de Sam Nzima ayant fait le tour du monde : celui de ces trois enfants fuyant la charge de la police sudafricaine, avec Hector Pieterson en victime expiatoire porté à bout de bras par Mbuyisa Makhubo (et à ses côtés sa soeur Antoinette Sithole). Cette image rappelle l'iconographie chrétienne de la pietà (Marshall, 2006) L'attraction du SOuth WEst TOwsnhip doit également beaucoup aux « héros » que sont Nelson et Winnie Mandela et secondairement à l'archevêque Desmond Tutu. Les symboles politiques et historiques de la nation arc-en-ciel sont ici rassemblés. À l'instar du musée de Robben island (Coombes, 2003), ces figures tutélaires surdéterminent la prestation, au risque de ne plus offrir une histoire complexe et totale de la lutte pour la libération. 
Illustration 3a - Rue Vilakazi et le Hector Pieterson Memorial

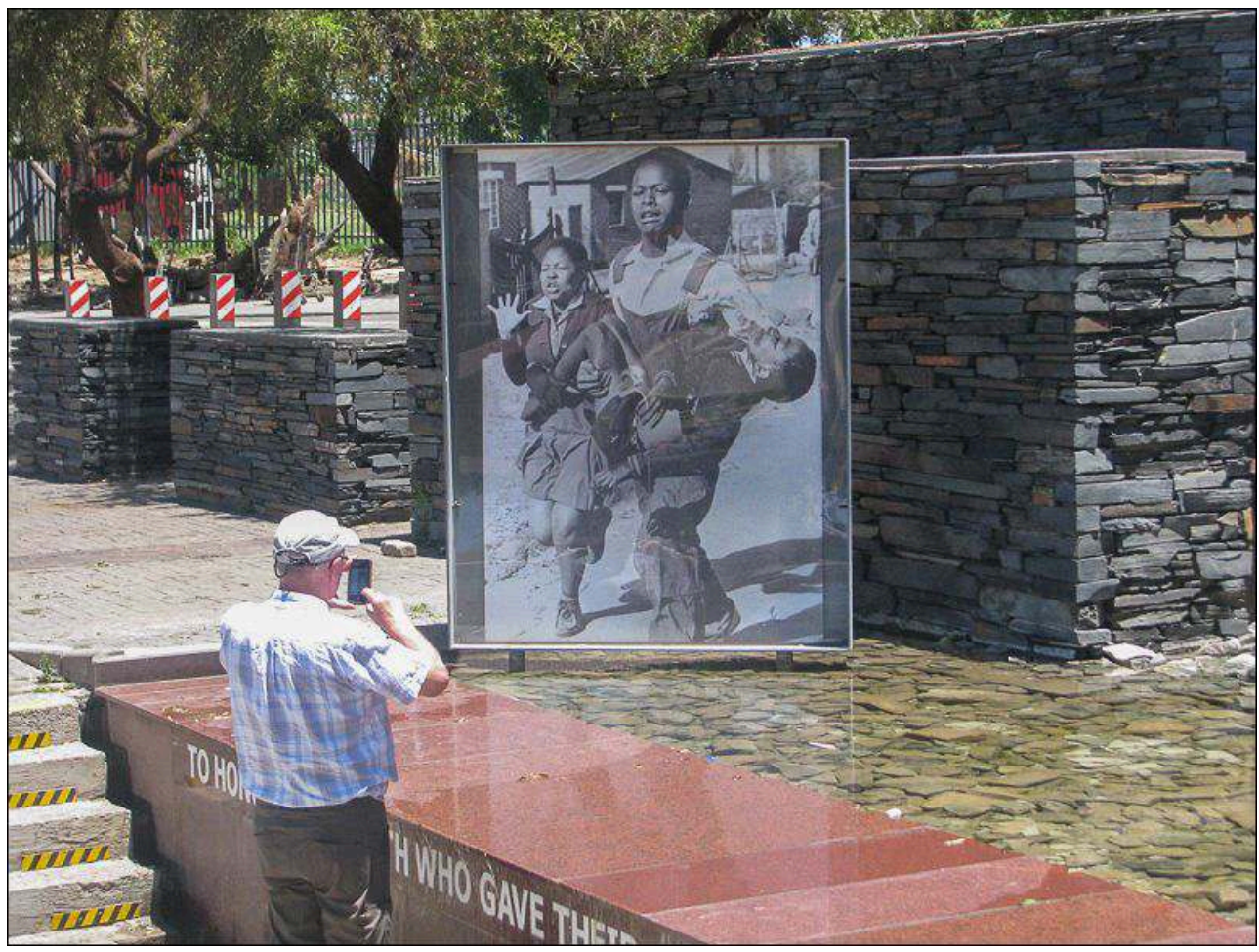

La principale artère des tours de Soweto est sans conteste cette rue avec au bout le Hector Pieterson Memorial, exposant son célèbre cliché de 1976.

Source : F. Folio, 2014 
Illustration 3b - Rue Vilakazi et la maison des époux Mandela

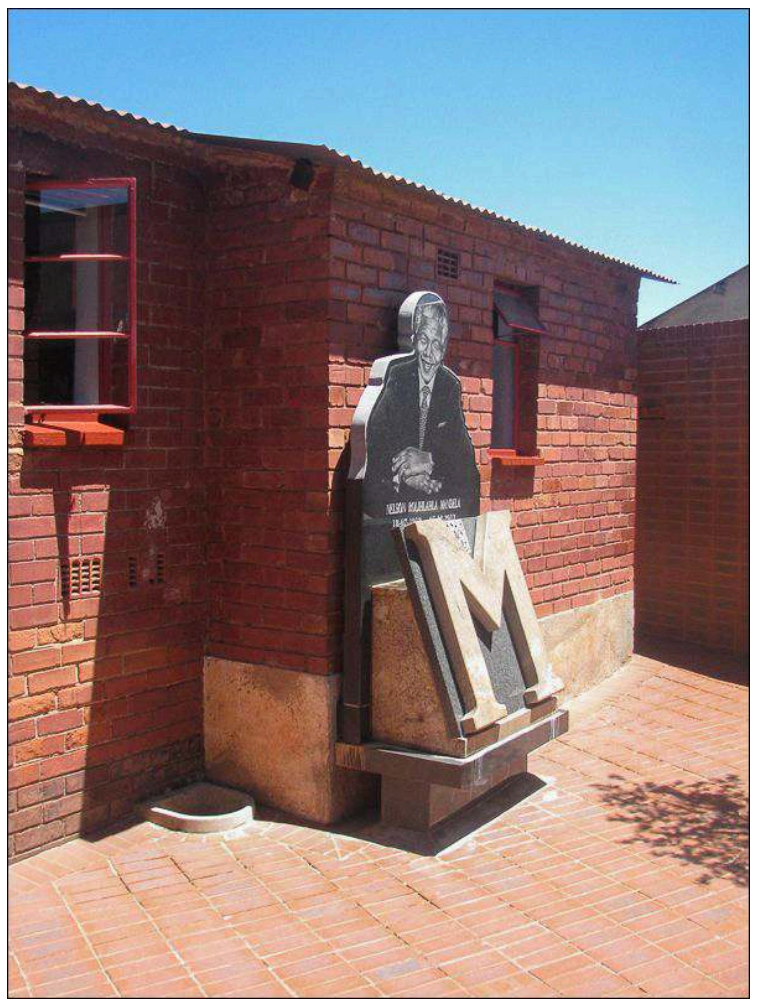

La rue abrite par ailleurs la maison des époux Mandela reconvertie en musée intimiste. Source : F. Folio, 2014 
Illustration 3c - Square Walter Sisulu

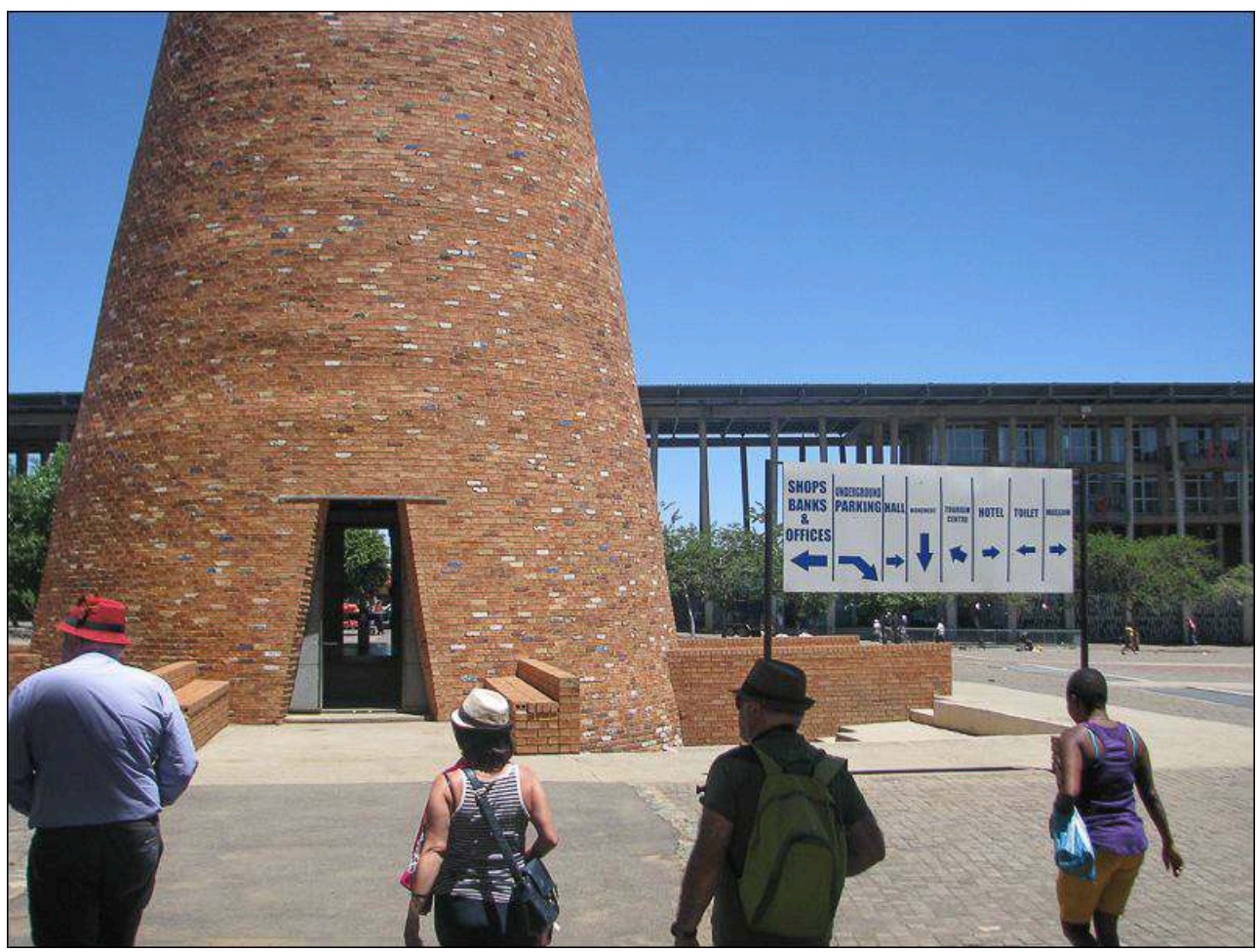

Le square est une autre des places réhabilitées de Soweto. Le lieu où fut signée la charte de la liberté par l'ANC a servi de prétexte à l'ouverture de banques et d'offices ...

Source : F. Folio, 2014. 


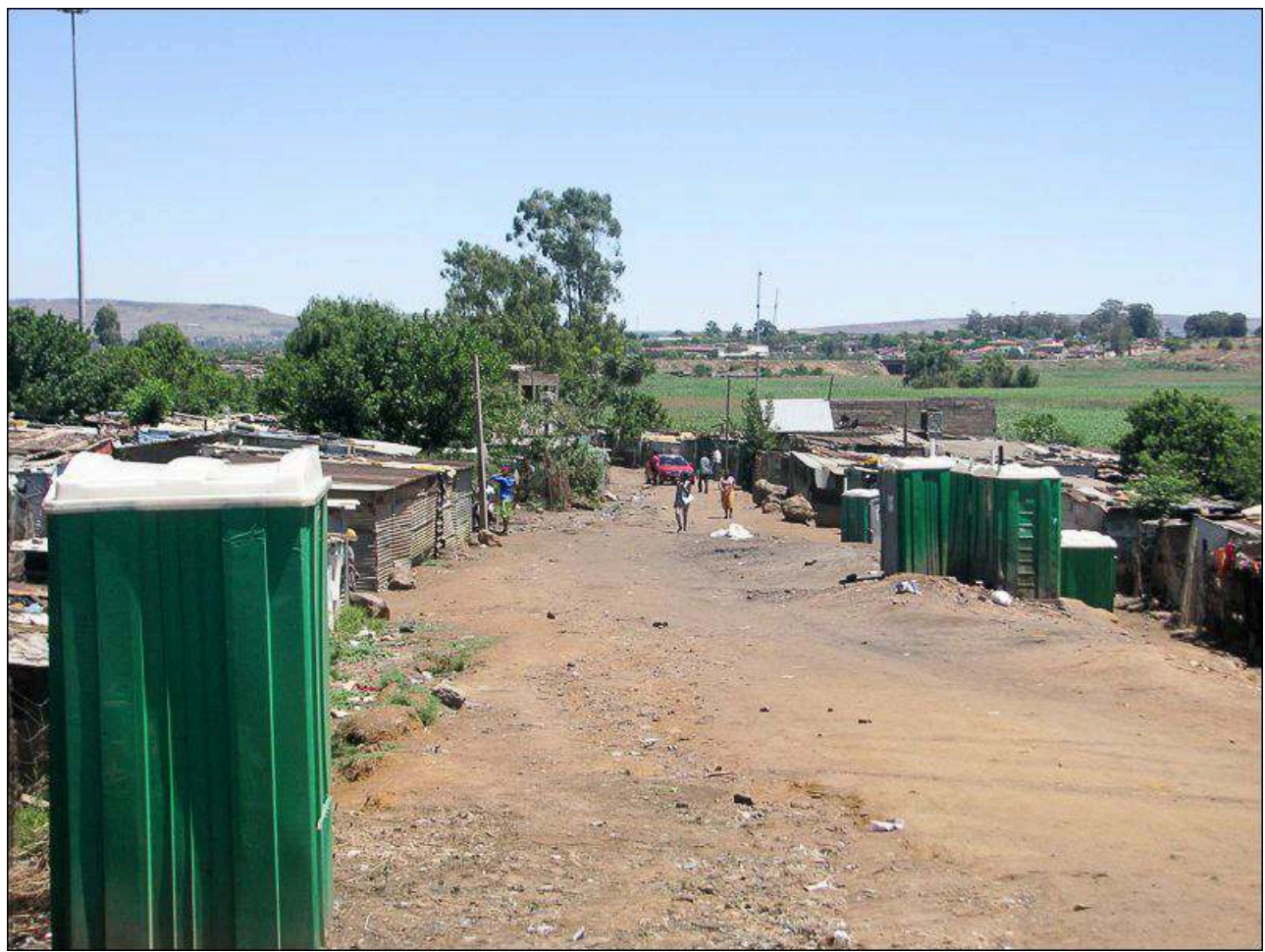

le tout aux abords d'un camp de squatters

Source : F. Folio, 2014

\section{D'une analyse de la demande du Dark tourism : l'écheveau des motivations}

\section{Une activité contemporaine devenue incontournable?}

Cette dernière partie de l'article se propose de présenter une synthèse critique sur les motivations inhérentes à ce type de tourisme en retournant à un cadre général (éclairé à ce stade par la "matière " sud-africaine). Les contours du tourisme dit sombre sont plus complexes que ce qui est généralement relayé dans les médias. Cela finit par mettre en débat la notion même. Le regard que lui portent les visiteurs est varié et très difficile à appréhender (Urry, 1990). De la catharsis au jeu de rôle par la mise en situation, peut-on parler de voyeurisme importun ou plutôt d'un néo tourisme emphatique et métonymique ? Si le Dark tourism correspond à toute forme de tourisme impliquant la visite de sites associés à la mort, à la tristesse ou à la destruction, beaucoup reste à faire pour cerner ses modalités, qu'elles soient relatives aux types de pratiques et aux sites concernés - nous avons sur ces deux points proposé quelques pistes de discussion au début de cet article -, ou encore aux motivations liminaires. La caractérisation de ce sous-secteur touristique et sa définition peuvent sembler éloignés de la réalité de voyage des individus. Mais dans les faits, une clientèle répond présente. Toutefois, il convient aussi de rester prudent et d'éviter les raccourcis et simplifications. 
Tout d'abord, si l'on s'en tient à l'expérience sud-africaine, on remarque que, bien que les sites liés au Dark tourism s'inscrivent désormais comme un produit signifiant, cela n'en fait pas encore, pour l'heure, la prestation numéro un recherchée en terre arc-enciel. L'analyse des statistiques nationales montre que les attractions patrimoniales relatives par exemple à l'apartheid incarnent plutôt un ajout aux séjours touristiques, motivés à la base par d'autres considérations ou motifs de voyage. Pour les touristes étrangers ultramarins (un des cœurs de cible du Dark tourism), la motivation première liée à un déplacement en Afrique du Sud reste et de loin la découverte de ses paysages naturels et surtout de sa faune. Cette découverte se déroule dans le cadre de safaris dans les nombreux parcs nationaux et réserves naturelles. L'expérience sud-africaine se compose d'un mélange de vie sauvage (en particulier l'appel des big five) et secondairement de ce que l'on pourrait appeler le «tribalisme vernaculaire ». Il s'agit de spectacles ethniques se déroulant dans des villages «traditionnels» (tels que Lesedi ou Shakaland...), associés aux populations noires de l'arrière-pays. Un examen plus attentif des sites sombres visités (dont certaines destinations mentionnées précédemment), révèle que leur proximité ou leur possibilité d'accès depuis les zones les plus recherchées du pays joue en leur faveur. Il s'agit pour ne prendre que deux exemples du Victoria \& Alfred Waterfront au Cap concernant Robben island ; à Jo'burg on citera le parc à thème de Gold Reef City pour le musée de l'apartheid. Parler d'espaces de confins touristifiés - une île au large, des quartiers périphériques ou dégradés... - est donc relatif, au vu de la forte accessibilité, du balisage et dorénavant de l'image (ou marquage) recodé du lieu. En fait tout se passe comme si ces prestations, du moins les plus prisées, agissaient en complément d'un séjour touristique global en Afrique du Sud. Elles bénéficient de leur nouveauté, de leur notoriété et d'un bon positionnement qui va venir enrichir l'éventail des pratiques. Mais ces prestations incarnent une pratique d'appoint; elles sont rarement le but premier du voyage (hormis un site comme Robben island $\left.{ }^{7}\right)$. Nous sommes bien là dans l'ère des " combinés touristiques ", associant de multiples expériences. Quant à la seconde clientèle du Dark tourism, c'est à dire les populations scolaires sud-africaines, les visites s'opèrent ici en groupe dans un cadre didactique et mémoriel, que l'on ne pourra que difficilement caractériser de «non-contraint ». Notons que ces excursions éducatives motivées par une institution sont particulièrement nombreuses en septembre, qui est le mois des commémorations du patrimoine en Afrique du Sud'.

Les finalités réelles des visiteurs sont à préciser plus finement car, en définitive, le Dark tourism reste un domaine d'étude assez nouveau. Selon Sharpley et Stone (2009), la littérature académique sur ce type de tourisme demeure éclectique et trop fragile d'un point de vue théorique. En conséquence, la compréhension exacte du phénomène demeure limitée. Selon ces mêmes auteurs, les motivations inhérentes restent sousévaluées. Plusieurs facteurs, d'ordre sociologique et psychologique, peuvent être mobilisés et certains sont controversés.

L'affirmation selon laquelle le Dark tourism serait une activité complètement nouvelle est un cliché solidement enraciné. La fascination pour la mort et les désastres ou drames n'est en fait pas si originale que cela. La nouveauté se situe plus dans le fait qu'elle s'enracine depuis une vingtaine d'années dans la bulle médiatique et dans l'industrie du tourisme 9 . Cependant, la curiosité motivant une venue sur des lieux tragiques remonte à fort loin. D'après Stone et Sharpley (2008), des sites tels que celui de la crucifixion du Christ ou encore des exécutions médiévales peuvent être 
considérés comme de très vieilles destinations «touristiques » liées à la mort! Des spectacles de gladiateurs dans l'antiquité (notamment au Colisée à Rome), à la "Caverne des Grands Voleurs ", précurseur de la chambre des horreurs de Marie Tussaud, on saisit que la souffrance et la mort sont depuis longtemps utilisées comme spectacle de divertissement. Selon Seaton (1996), la tradition thanatoptique est bien antérieure à son incorporation dans la bulle touristique moderne. En effet, elle composait une part de la réflexion morale sur la spiritualité et les pensées philosophiques, notamment occidentales, caractéristique de la période romantique de la fin du XVIII ${ }^{\mathrm{e}}$ siècle et du début du XIX ${ }^{\mathrm{e}}$. Ainsi, pour Seaton, le site de Waterloo à partir de 1816 et les déambulations à Pompéi sont les plus évidentes destinations thanatoptiques de l'ère romantique. La connexion entre la violence et le divertissement ne serait finalement que le versant extrême d'une forme, plus moralement acceptable, d'attirance pour le dramatique. L'évocation d'un mélange d'émotions mêlant attraction et dégout, effroi et frissons, à travers tout ce qui a trait à la tragédie n'est donc pas qu'une question de sites touristiques, bien qu'elle se standardise en prestations et en produits. Elle est aussi présente dans la littérature, le monde de la musique, l'art et bien évidemment la télévision ou le grand écran. L'association du tourisme patrimonial avec les atrocités de l'histoire n'a fait que suivre une voie qu'ont empruntée avant elle d'autres supports et objets, avec les mêmes ressorts sous-jacents, les mêmes interrogations morales et les mêmes débats éthiques ${ }^{10}$. L'être humain n'a-t-il pas toujours été intrigué par la mort et la souffrance (même s'il s'en défend parfois) ? Ne faudrait-il pas y voir, a minima, un essai de compréhension et de rationalisation de sa destinée et de son trépas, pris dans un sens général?

\section{Face aux multiples ressorts : convier les sciences sociales}

41 Certains auteurs s'accordent à dire qu'un touriste «sombre » est celui pour qui les attraits liés au tragique et au drame doivent être les premières motivations du voyage. Ce sont ces mêmes facteurs qui distingueraient le Dark tourism du tourisme de mémoire, qui ferait lui plutôt appel à l'intérêt pour l'histoire. Toutefois la limite devient rapidement ténue ${ }^{11}$. Selon nombre de Nord-Américains, se rendre sur le site de Ground Zero est un acte patriotique que tous les ressortissants nationaux devraient accomplir. On aborde là un autre aspect qui concerne la fierté nationale. Des survivants ont parfois l'irrépressible envie/besoin de revenir sur un lieu de drame (Smith, 1996). Certains épisodes peuvent susciter chez les descendants une envie de s'y recueillir pour se (re)construire. Démarches qui relèvent de la recherche du patrimoine personnel et de la quête de ses racines (Mowatt, Chancellor, 2011). Le succès de la maison des Esclaves sur l'île de Gorée dans la baie de Dakar est un célèbre exemple de Dark tourism. La mauvaise conscience et le désir de rédemption peuvent aussi être des éléments mobilisateurs lorsque l'on touche à des soubresauts de l'histoire (la traitre, l'esclavage, la colonisation, les génocides...). Quant aux catastrophes récentes, elles bénéficient, en tant que produit d'appel, d'un impact médiatique et elles nourrissent une part d'anxiété et de doute face aux effets de la modernité (Lennon, Foley 2000). De surcroît, on ne peut évacuer la quête d'expériences nouvelles et « déroutinières » car c'est là une réalité du tourisme contemporain. Il existe de nos jours un public simplement à l'affut d'une exclusivité touristique permanente (Mayo and Jarvis, 1981). Qui plus est, si certaines personnes recherchent parfois des lieux qui inspirent le respect et le souvenir, l'éducation et une forme d'empathie et de responsabilisation sociale (Hyde \& 
Harman, 2011), d'autres éprouvent un besoin de voir ces endroits pour s'autoconvaincre de leur réalité tragique («see it to believe it ", Biran et al, 2011). D’autres encore, et il ne faut pas le nier, retirent du plaisir à scruter un objet macabre pour ce qu'il est (Lennon, Foley, 2000). On formule ce dernier sentiment par l'expression "Schadenfreude», proche du sadisme, qui est une excitation maligne induite par le malheur d'autrui. Enfin, pour certains individus, le Dark tourism représente un moyen de méditer sur leur propre mortalité et sur leur réaction imaginée/fantasmée par rapport à cela. On peut parler à ce niveau de "transfert métonymique » : qu'aurai-je fait si ça avait été moi ? Si j'avais été présent ? À la place de la victime... ou du bourreau le cas échéant?

Les définitions reconnues du tourisme sombre s'avèrent par conséquent très générales, qu'elles soient de Lennon \& Foley (2000) ou de Dann \& Seaton (2003). Elles s'inscrivent dans la tendance académique récente d'identifier et de labelliser les nouvelles formes de tourisme et de subdiviser le secteur en autant de niches (Novelli, 2005). À partir des travaux scientifiques sur le sujet, le tableau 3 ci-dessous propose au moins six facteurs mobilisateurs du Dark Tourism, pouvant eux-mêmes se combiner ou agir indépendamment.

Tableau 3 - Essai de typologie des motivations du Dark tourism

\begin{tabular}{|l|l|l|}
\hline 1 & $\begin{array}{l}\text { Un attrait pour la connaissance et la compréhension de } \\
\text { l'histoire. }\end{array}$ & « édutourisme » \\
\hline 2 & $\begin{array}{l}\text { Une mission davantage sociale et de responsabilité afin de (se) } \\
\text { sensibiliser pour éviter que cela ne se reproduise }\end{array}$ & $\begin{array}{l}\text { «never again tourisme » ou le } \\
\text { devoir de mémoire }\end{array}$ \\
\hline 3 & Une quête d'identité, un retour aux sources & «tourisme des racines » \\
\hline 4 & La motivation de pèlerinage, un sentiment de repentance & «mea culpa tourisme » \\
\hline 5 & Une envie de découvrir une offre exclusive et incongrue & «tourisme de curiosité » \\
\hline 6 & $\begin{array}{l}\text { Une attirance moins avouable pour la violence et la } \\
\text { souffrance en tant que telle }\end{array}$ & $\begin{array}{l}\text { «tourisme sadomasochiste et } \\
\text { pornographique » }\end{array}$ \\
\hline
\end{tabular}

Cette synthèse s'appuie sur les écrits de Tarlow (2005), de Ashworth (2004) et de Dann (2005).

43 Le spectre des motivations est très large et c'est pourquoi il faut éviter tout jugement moralisateur hâtif sur les clientèles qui s'adonnent au Thanatourisme. Ce qui n'empêche pas un examen critique de l'appellation générique de Dark tourism, à l'instar de ce qui est proposé par Bowman et Pezzullo (2010). D’une part, nous l'avons dit, il semble trop englobant et simplificateur. D'autre part, il est connoté négativement. Derrière l'ensemble des définitions proposées, les mots sont forts et guère amènes. Qualifier un site comme « Dark » ou sombre revient parfois à entrer en contradiction avec les messages que les opérateurs ambitionnent de porter. Car le discours mémoriel à vertu pédagogique devient de plus en plus inéluctable (très probablement aussi pour légitimer la prestation et en assurer la réussite !) ${ }^{12}$. Le cas sudafricain est symptomatique du souhait de délivrer un message universel, celui de la résistance à la politique d'oppression, celui de la réconciliation et de la reconstruction 
nationale (Folio, 2010). Les tours du township de Soweto comme les expériences carcérales - cf. Kapstein, 2009 pour le cas de Robben island - ne sont pas appréciés parce ce qu'ils incarneraient des lieux de refoulement (ou d'incarcération) rudes et extrêmes, mais parce qu'ils représentent la patrimonialisation d'endroits insolites où dominaient des valeurs telles que l'abnégation et le courage. Plutôt que le «Dark tourism ", une autre forme de tourisme est évoquée localement, le « Hope tourism ». Et cela semble fonctionner. Selon les enquêtes de T. Mudzanani (2014) initiées au Mémorial Hector Pieterson, (entretiens non directifs), la nostalgie (" l'effet Mandela »), l'aspect pédagogique (s'instruire, apprendre de ce tournant historique) et la couverture médiatique (presse, roman, cinéma etc.) figurent parmi les motifs avancés par les participants du tour. Même si un attrait singulier pour la «dangerosité »- au vue de l'image marquée de Soweto - et pour la montée d'adrénaline ne sont pas à exclure, les principaux aspects mis en avant concernent en outre : la nouveauté (l'envie d'accéder à une prestation unique et originale) ; le désir de s'éloigner de la routine, du quotidien; enfin le souhait de nouer de nouvelles relations sociales. Les discours ambiants sont donc éclectiques et reflètent bien les préoccupations de notre époque. Ils ont une visée assez humaniste et optimiste, et ne sont nullement réducteurs et funestes.

Incontestablement, des aspects sociologiques et psychologiques, parfois antagonistes et browniens, demeurent ancrés en chacun de nous dans la pratique du Dark tourism (Rojek, 1997). Nous en évoquerons trois, qui mériteraient d'être approfondis et qui participent de l'attrait putatif pour ce phénomène très complexe.

- En premier lieu, l'expérience sensorielle est incomparable par rapport aux autres supports que ce soit les lectures ou les visionnages - de matériaux historiques a fortiori tragiques. L'intensité perçue ne sera jamais la même (Graham, Howard, 2008). Être sur place, ressentir, vivre le lieu, permet de mieux le saisir et en parler. Être présent, c'est aussi en retirer un objet-souvenir, caractéristique d'une époque tout à la fois hédoniste et portée sur le relationnel via les réseaux sociaux, dont les égoportraits en sont la forme la plus poussée (Gunthert, 2015). C'est probablement en cela que le tourisme moderne mondialisé et dérégulé, baignant dans une ère décomplexée et hyperconnectée, amplifie techniquement et plus aisément cette dynamique, en multipliant le champ des possibles.

- En second lieu à propos de la limite entre mémoriel et voyeurisme, A. Tezenas (en parlant des genocide tours au Rwanda), indique qu'il s'agit «certes de se recueillir devant les victimes, mais aussi de revivre l'histoire» (in Bossard, 2013). Selon lui « les gens veulent se sentir vivants face à tous ces morts ». Finalement, cela ne leur permet-il pas de relativiser leurs problèmes et soucis, l'éventuelle tiédeur ou banalité de leur existence? Ne peut-on considérer qu'ils accèdent, à ce moment, à une parcelle de réponse dans leur quête de soi et de sens dans un monde vu comme uniformisé voire aseptisé ? La charge émotionnelle de ces tours inciterait, par un mouvement de balancier, à finalement se satisfaire de ce que l'on a et de ce que l'on est. Il y a là un phénomène de retour sur l'essentiel qui s'opérerait.

- En dernier lieu et comme l'explique John Lennon de l'université de Glasgow, certains lieux du crime nous troublent parce qu'ils sont aussi directement liés à notre propre capacité de tuer ou de dominer! Ce type de tourisme, du moins dans les cas où une responsabilité humaine est engagée, nous renvoie donc, par là-même, à nos faiblesses animales, à notre capacité d'autodestruction en tant qu'être humain (Homo homini lupus: "L'homme est un loup pour l'homme»), avec de plus une histoire qui semble bégayer et se répéter. Cependant, pour Benjamin (1969), il n'existe pas de trace de civilisation qui n'évoque pas, dans le même temps, des traces de barbarisme. Et l'inverse est tout aussi vrai : tout moment d'histoire lié au barbarisme est un document éclairant sur la résistance et la résilience. En 
nous renvoyant parfois à ce qu'il y a de plus extrêmes en nous, le Dark tourism peut provoquer un effet catharsis avec un retour à une normalité, mais une normalité rassurante et « consciente» (ou éclairée).

\section{Conclusion}

45 loisirs d'autre part pourrait sembler contre nature. Elle suscite des débats académiques éthiques contemporains (Rojek, 1993 ; Tunbridge et Ashworth, 1996). Certains auteurs dénoncent le caractère parfois "folklorisant ", banalisant et quelquefois même nonauthentique d'un mariage qui peut en définitive s'avérer inconvenant (Walsh 1992 ; Urry, 1995). Macdonald (1997) pour sa part, insiste sur une distinction qu'il conviendrait d'établir entre ce qui est, d'un côté du ressort du sensationnel, de la fiction et du divertissement pur, et de l'autre d'une histoire authentique, pudique et formatrice, incitant le public à être attentif en amont et à prêter une attention particulière aux intentions et aux finalités des acteurs en charge de la prestation. De sorte qu'une tendance semble se dégager. Se remémorer le passé et apprendre de celuici apparait être la nouvelle doxa des prestations du tourisme sombre ou thanatourisme. Le but recherché est de rendre hommage aux victimes et d'aider à la reconstruction locale dans les cas de catastrophes récentes, ainsi que d'initier une réflexion tournée sur soi-même. Il s'agit d'apprendre de cette histoire tourmentée difficile et de la réalité du monde, de notre planète et de ses excès, ainsi que des travers de la nature humaine. Ce qui revient à se confronter à une certaine vérité et à une radicalité. Le Dark tourism est essentiellement un phénomène béhavioriste (Seaton, 1996).

Ce patrimoine historique grave devenue matière touristique et donc économique incarne, dans ce contexte, la face dissonante de ce qui est communément exposé dans le secteur du tourisme: soit un monde heureux, généreux et bienveillant. Ce qui est original dans le succès de ces tours, c'est que le tourisme, vu habituellement comme une échappatoire insouciante à un quotidien stressant et répétitif, « capte » dorénavant des visiteurs qui sont prêts à ajouter ces prestations difficiles à leur programme ou combiné touristique. Même si beaucoup de touristes accèderont à ce type de prestations en complément du tour/séjour initial, avant généralement un retour revigorant à la normalité et au confort du quotidien. Mais quoi qu'il en soit, l'évolution de «l'homo touristicus, » dont la demande est beaucoup moins évidente et prévisible qu'on ne pourrait le croire, doit être étudiée. Le Dark tourism est ainsi devenu un champ de recherches multidisciplinaires original à approfondir, dans ses modalités comme dans ses enjeux.

Afin d'éviter tout oubli et négationnisme, le rôle crucial de la " mémorialisation » est à souligner. À l'instar de ce qu'a pu décrire K. Till sur Berlin, le cas des prestations de la métropole de Johannesburg est finalement très emblématique, en qualité de portedrapeau du passé urbain sud-africain, de ce processus en cours, ce que les anglophones appellent l'effacement (erasure) des histoires subalternes. En Afrique du Sud, cette «mémorialisation » du passé (Grunebaum, 2011 ; Dubin 2006) profite à divers acteurs (secteur privé, communautés, groupes d'intérêt...), tout en étant adossée à un agenda éminemment politique (Marshall, 2010, 2009). En effet, elle convie stratégiquement développement local, renforcement de l'appropriation et du lien communautaire et réécriture du récit national. Comme le rappelle M. Houssay-Holzschuch (2010), « La 
mémoire et les politiques mémorielles sont donc un problème crucial et des espaces spécifiques (...) sont classiquement instrumentalisés pour mettre en place une identité proprement sud-africaine $»$.

Cependant le segment du tourisme sombre n'évite pas toujours un écueil : celui de la visite des monuments souvent présentée comme très singulière et sans comparaison. Elle incarnerait le contre-exemple inédit des prestations touristiques classiques. Or, à y regarder de plus près, les plus fameux sites du thanatourisme sont eux-mêmes progressivement intégrés puis standardisés par l'industrie du tourisme. Outre les aspects organisationnels habituels (sécurité, confort...), un risque d'ordre " mémoriel » peut survenir. C'est pourquoi, il faut d'une part, veiller à ce que la normalisation de ce type de prestations ne se limite pas à la retranscription d'une histoire évènementielle et simplifiée (une « histoire mainstream » ou du courant dominant); d'autre part il ne faudrait pas non plus qu'elle sombre dans un passé revisité qui deviendrait romanesque, d'obédience uniquement optimiste et ramené au courage ou au combat de quelques individualités ou icones « identifiantes », fussent-elles estimables.

\section{BIBLIOGRAPHIE}

Ashworth G.J., 2002. Holocaust tourism: The experience of Krakow-Kazimierz. International Research in Geographical and Environmental Education, 11(4), p. 363-367.

Ashworth G.J., 2004. Tourism and the heritage of atrocity: Managing the heritage of South African apartheid for entertainment. In Singh T.V., Tourism seeks new horizons. CABI Basingstoke. University of Groningen, NL.

Benjamin W, 1969. Illuminations. Ed. Hannah Arendt, trans. Harry Zohn, New York, SchockenBooks.

Biran A., Poria Y., Oren G., 2011. Sought Experience at Dark Heritage sites. Annals of Tourism Research, Vol. 38 (3), p. 820-841.

Blom T., 2000. Morbid tourism: a postmodern market niche with an example from Althorp. Norsk Geografisk Tidsskrif, 54(1), p. 29-36.

Bossard C., 2013. Entre génocides et tsunamis le tourisme noir a ses adeptes. La nouvelle république.fr, 10/08/2013. http://www.lanouvellerepublique.fr/France-Monde/Actualite/24Heures/n/Contenus/Articles/2013/08/10/Entre-genocides-et-tsunamis-le-tourisme-noir-a-sesadeptes- 1574530

Bowman M. S., Pezzullo P. C., 2010. What's so ‘Dark' about 'Dark Tourism'? Death, Tours, and Performance ». Tourist Studies, 9 (3), p. 187-202.

Bremner L., 2007. Memory, Nation-Building and the Post-Apartheid City. The Apartheid Museum in Johannesburg. In N. Murray, N. Shepherd, M. Hall, Desire lines : space, memory and identity in the post-apartheid city. Eds Routledge, New York, p. 85-103.

Coombes A. E., 2003. History after apartheid. Visual Culture and Public Memory in a Democratic South Africa. Witwatersrand University Press, Johannesburg. 
Coldwell W., 2013. Dark tourism: why murder sites and disaster zones are proving popular. The Guardian, Thursday 31 October. http://www.theguardian.com/travel/2013/oct/31/dark-tourismmurder-sites-disaster-zones

Constitution Hill Foundation, 2006. Number 4: The Making of Constitution Hill. Penguin Group (USA) Incorporated.

D’Angelo A., 2014. Record tourists boost Cape Town. Business report, IOL, January 27 2014. http:// www.iol.co.za/business/news/record-tourists-boost-cape-town-1.1637105\#.VTCiR8aolGg

Dann G.M.S, 2005. Children of the dark. In G. J. Ashworth, R. Hartmann (Eds.), Horror and human tragedy revisited: The management of site of atrocities for tourism. UK, Cognizant. p. 233-252.

Dann G.M.S., 1998. The dark side of tourism. Études et Rapports, Série L. Aix-en-Provence, Centre International de Recherches et d'études touristiques.

Dann G.M.S., Seaton A.V., (eds), 2003. Slavery, contested heritage and thanatourism. Haworth New York /London.

Dewailly J-M., 2006. Tourisme et Géographie, entre pérégrinité et chaos. L'Harmattan, Tourismes et Sociétés, $221 \mathrm{p}$.

Dubin S. C., 2006. Transforming museums: mounting Queen Victoria in a democratic South Africa. Palgrave Macmillan, New York.

Dunkley R., 2005. Tourism Society Seminar Event 'Dark Tourism - Cashing In On Tragedy?'. Kensington Close Hotel, London, 17th October 2005.

Fassin D., 2007. «Ce qui s'est vraiment passé » L'expérience du musée de l'Apartheid. Revue d'Anthropologie et d'Histoires des Arts, $n^{\circ}$ 5, Sismographie des terreurs, p. 52-61.

Findley L, 2005. Building change: architecture, politics and cultural agency. London: Routledge.

Folio F., 2014. Patrimonialisation et (re)valorisation touristiques dans la métropole d'eThekwini (KwaZulu-Natal, Afrique du Sud) : à la croisée des enjeux politiques et économiques. Cybergeo : European Journal of Geography [En ligne], Espace, Société, Territoire, document 666, mis en ligne le 07 février 2014, consulté le 10 août 2015. http://cybergeo.revues.org/26174 ; DOI : 10.4000/ cybergeo. 26174

Folio F., 2010. Les Township tours au KwaZulu-Natal (Afrique du Sud) : d'une réappropriation historique et identitaire à l'avènement d'un socio-tourisme. EchoGéo [En ligne], Sur le Vif, mis en ligne le 09 décembre 2010, consulté le 06 août 2015. http://echogeo.revues.org/12243 ; DOI : 10.4000 /echogeo. 12243

Graham B., Howard P., 2008. Heritage and Identity. In B. Graham, P. Howard (Eds.), The Ashgate Research Companion to Heritage and Identity. England, Ashgate Publishing Ltd.

Grunebaum H., 2011. Memorializing the Past: Everyday Life in South Africa after the Truth and Reconciliation Commission. Transaction, Publishers, $247 \mathrm{p}$.

Guinard P., 2011. L'art public de l'apartheid à Johannesburg, un patrimoine ? Le cas de la statue de Carl von Brandis. Géographie et cultures, [Online], 79 | 2011, Online since 25 February 2013, connection on 30 July 2015. http://gc.revues.org/388 ; DOI : 10.4000/gc.388

Hernandez, J., 2008. Le tourisme macabre à La Nouvelle-Orléans après Katrina : résilience et mémorialisation des espaces affectés par des catastrophes majeures. Norois, 208|2008, p. 61-73. Hewison R., 1987. The Heritage Industry. London, Methuen. 
Houssay-Holzschuch M., 2010. Crossing boundaries: vivre ensemble dans l'Afrique du Sud postapartheid. HDR, Université de Paris I Panthéon-Sorbonne.

Hyde K., Harman S., 2011. Motives for a secular pilgrimage to the Gallipoli battlefields. Tourism Management, 32(6), p. 1 343-1 351.

Iribarnegaray L., 2015. André Gunthert : « Embrayeur de conversation, le selfie doit être moche ». Libération, 5 août 2015.

Jamieson W., 2006. Community destination management in developing economies. New York, Haworth Hospitality.

Kang E-J., Noel Scott S., Jeonglyeol, Lee T., Ballantyne R., 2012. Benefits of visiting a "dark tourism" site: the case of the Jeju. April 3rd Peace Park, Korea. In Tourism management : research, policies, practice, Amsterdam - Vol. 33.2012, 2, p. 257-265.

Kapstein H., 2009. A Travel Paradise: Tourism Narratives of Robben Island. Safundi: The Journal of South African and American Studies, $\mathrm{n}^{\circ} 10, \mathrm{p}$ 449-458.

Kendle A., 2008. Dark Tourism: A Fine Line Between Curiousity and Exploitation. Vagabondish, January 24, 2008. http://www.vagabondish.com/dark-tourism-travel-tours/

Kirshenblatt-Gimblett B., 1998. Destination Culture: Tourism, Museums, and Heritage. Berkeley, University of California Press.

Knafou R., 2012. Les lieux du voyage. Paris, Le cavalier Bleu Éditions, 215 p.

Khumalo T., Sebatlelo P., van der Merwe C. D., 2014. 'Who is a heritage tourist?' a comparative study of Constitution Hill and the Hector Pieterson Memorial and Museum, Johannesburg, South Africa. African Journal of Hospitality, Tourism and Leisure, Vol. 3 (1).

Lennon J., Foley M., 2000. Dark tourism: the attraction of death and disaster. New York, TJ International.

Lennon J., Foley M., 1996. Dark Tourism. London, Continuum.

MacCannell D., 1992. Empty Meeting Grounds. London, Routledge.

Macdonald S., 2009. Difficult heritage: Negotiating the Nazi past in Nuremburg and beyond. London, Routledge.

Macdonald S., 1997. A People's Story: Heritage, Identity, and Authenticity. In C. Rojek , J. Urry, eds., Touring Cultures. London, Routledge, p. 155-176.

Marschall S., 2010. The Memory of Trauma and Resistance: Public Memorialization and Democracy in Post-apartheid South Africa and Beyond. Safundi: The Journal of South African and American Studies, $\mathrm{n}^{\circ}$ 11, p 361-381.

Marschall S., 2009. Landscape of memory: commemorative monuments, memorials and public statuary in post-apartheid South-Africa. Brill.

Marschall S., 2006. Visualizing Memories: The Hector Pieterson Memorial in Soweto. Visual Anthropology, n 19, p. 145-169.

Mayo E., Jarvis L., 1981. The Psychology of Leisure Travel: Effective Marketing and Selling of Travel Services. Boston, CBI Publishing Co., Inc.

Mowatt R.A., Chancellor C.H., 2011. Visiting death and life: Dark tourism and slave castles. Annals of Tourism Research, 38(4), p. 1410-1 434. 
Mudzanani T., 2014. Why is Death so Attractive? An Analysis of Tourists' Motives for Visiting the Hector Peterson Memorial and Museum in South Africa. Mediterranean Journal of Social Sciences, vol. 5, n 15, MCSER Publishing, Rome-Italy July 2014.

Novelli M., 2005. Niche tourism: contemporary issues, trends and case. Oxford: ButterworthHeinemann.

Nuttall S., Coetzee C., 1998. Negotiating the past: the making of memory in South Africa. Oxford University Press, Cape Town.

O'Neill S., 2002. Soham pleads with trippers to stay away. Daily Telegraph; http:// www.telegraph.co.uk/news/main.jhtml?xml=/news/2002/08/26/nfen26.xml\&amp;sSheet=/ portal/2002/08/26/ixport.html, accessed 13 March 2008.

Poon A., 1993. Tourism, technology and competitive strategies. Oxford, CAB, Wallingford.

Rogerson C, 2006. Pro-Poor local economic development in South Africa: The role of pro-poor tourism. Local Environment, 11 (1), January 2006, p. 37-60.

Rogerson C.M., Visser G. (eds), 2004. Tourism and development issues in contemporary South Africa. Pretoria: Africa Institute of South Africa.

Rojek C., 1993. Ways of Seeing-Modern Transformations in Leisure and Travel. London, Macmillan. Rojek C., 1997. Indexing, dragging and the social construction of tourist sights. In C. Rojeck, J. Urry (eds), Touring Cultures: Transformations of Travel and Theory. London, Routledge, p. 52-74.

Samarbakhsh-Liberge L., 2000. L'African Renaissance en Afrique du Sud. De l'utilité ou de l'utilisation de l'histoire. In F-X. Fauvelle-Aymar, J-P. Chrétien et C-H. Perrot éds, Afrocentrismes. L'histoire des Africains entre égypte et Amérique. Paris, Karthala, p. 381-400.

Seaton A., 1996. Guided by the dark: from thanatopsis to thanatourism. International Journal of Heritage Studies, (2), p. 234-244.

Seaton A.V., 1999. War and thanatourism: Waterloo 1815-1914. Annals of Tourism Research, (26), p. $130-159$.

Seaton A.V, Lennon J.J, 2004. Moral panics, ulterior motives and alterior desires: thanatourism in the early 21st century. In Singh, TV(ed.), New horizons in tourism, Cambridge: CABI Publishing.

Sharpley R, 2005. Travels to the Edge of Darkness: Towards a Typology of Dark Tourism. In C. Ryan, S. Page and M. Aitken (eds), Taking Tourism to the Limits: Issues,Concepts and Managerial Perspectives. Oxford, Elsevier, p. 217-228.

Sharpley R, 2009. Shedding Light on Dark Tourism: An introduction. In Sharpley R., Stone P. (eds), 2009. The darker side of travel: the theory and practice of dark tourism. Bristol: Channel View, p. 3-22.

Sharpley R., Stone P., (eds), 2009. The darker side of travel: the theory and practice of dark tourism. Bristol, Channel View.

Smith, V.L., 1996. War and its tourist attraction. In A. Pizam, Y. Mansfeld (eds.), Tourism, crime and international security issues. New York, Wiley.

Stone P.R., 2006. A Dark Tourism Spectrum: towards a typology of macabre related tourist sites, attractions, and exhibitions. Tourism: An Interdisciplinary International Journal, vol. 54, $\mathrm{n}^{\circ} 2$, p. $145-160$.

Stone P., Sharpley R., 2008. Consuming dark tourism: a thanatological perspective. Annals of Tourism Research (35), p. 574-595. 
Strange C., Kempa M., 2003. Shades of Dark Tourism Alcatraz and Robben Island. Annals of Tourism Research, vol. 30, $\mathrm{n}^{\circ}$ 2, Elsevier Science Ltd., p. 386-405.

Swarns R.L., 2001. Oppression in Black and White: South Africa Museum Recreates Apartheid. New York Times, 10 December.

Tarlow P., 2005. The appealing dark side of tourism and more. In M. Novelli (Ed.), Niche tourism: Contemporary issues, trends and cases, London, Elsevier, p. 47-58.

Till K., 2005. The New Berlin: Memory, Politics, Place Paperback. University of Minnesota Press. 296 p.

Timothy D.J., 2011. Cultural heritage and tourism: an introduction. Ontario, Channel View.

Tunbridge J.E., Ashworth G.J., 1996. Dissonant Heritage - The Management of the Past as a Resource in Conflict. New York, John Wiley \& Sons.

Urbain J-D., 2011. L'envie du Monde. Paris, Editions Bréal, 269 p.

Urry J., 1990. The Tourist Gaze. London, Sage.

Walsh J., 1992. The Representation of the Past. London, Routledge.

White L, Frew E, 2013. Dark tourism and place identity: managing and interpreting dark places. New York, Routledge.

\section{NOTES}

1. Dans cet article, nous userons d'une analyse conceptuelle, agrémentée d'exemples empiriques. Nous croiserons, aux données académiques sur le sujet (ouvrages et articles scientifiques), un travail de terrain associant visites des sites abordés, entretiens auprès de personnes ressources (personnels administratifs, touristes, guides...) et lecture de la presse internet. Pour celle-ci, outre les grands journaux français et anglophones, nous avons accordé une attention particulière à la plateforme sud-africaine de médias en ligne iol.co.za (un groupe de presse indépendant publiant une quinzaine de grands journaux nationaux comme régionaux).

2. De Thanatos qui dans la mythologie grecque incarne la personnification de la mort.

3. Rappelons quelques éléments contextuels du tourisme en ce pays (Folio, 2014). Sur les 9,9 millions de visiteurs internationaux accueillis en 2010, 71,1\% étaient issus du continent et tout particulièrement - pour $68,9 \%$ d'entre eux - de la SADC (South African Development Community - organisation économique régionale rassemblant les pays d'Afrique australe). L'autre partie du marché est considérée comme "ultramarine » : elle compte pour $27,5 \%$ de la clientèle internationale, parmi laquelle $16,5 \%$ d'Européens et 4,2\% de Nord-américains. Une forte progression du marché des émergents est toutefois observée ces dernières années. Si les vacances sont l'apanage du marché ultramarin (pour plus de $50 \%$ des Américains, Européens et Asiatiques contre $12 \%$ des Africains), du côté des continentaux, c'est la motivation affinitaire (à $27 \%$ ) et le shopping (à 33\%) qui l'emportent. Le fait qu'existe également en Afrique du Sud une clientèle domestique importante, que l'on peut relier à des pratiques précoces, en particulier dans le cadre des sorties et voyages scolaires est aussi un élément intéressant. Le pays a une tradition récréative ancienne et assez remarquable pour l'Afrique sub-saharienne. Toutes les communautés sont aujourd'hui concernées. Au total 13,4 millions de vacanciers originaires d'Afrique du Sud ont été référencés en 2010 (pour 29,7 millions de séjours sur le territoire).

4. Il faut noter que ces musées, aux opérateurs et groupes d'intérêt différents, sont de statuts divers : Bo Kaap date de l'ère d'apartheid ; Robben Island est national ; le District Six fut constitué par les communautés ; quant à l'Apartheid Museum, on le verra, il fut créé par des acteurs privés dans un esprit résolument pragmatique. 
5. Défini comme un patrimoine discordant, en désaccord ou manquant de cohérence par rapport à un contexte social, spatial et/ou historique, par Tunbridge et Ashworth, 1996.

6. On se réfèrera ici aux mots de P. Stone : ceux de la « commercialisation de la mort ». Dans un article publié par le Guardian (Coldwell, 2013), le directeur exécutif de l'Institut pour la Recherche en Dark Tourism cite l'exemple du site du crash du Vol 93 le 9/2001 en Pennsylvanie. Dans un premier temps, ce sont les fermiers locaux qui ont pris conscience du potentiel du site en vendant des tours qu'ils proposaient eux-mêmes. Le succès venant, très vite de véritables opérateurs dont l'objectif est la recherche de profit sont venus occuper puis saturer le marché local, en en faisant cette fois une destination formelle appuyée par un mémorial.

7. Et là encore il faut le nuancer: pourtant classé à l'UNESCO depuis 1999, Robben island n'accueille «qu »'un peu plus de 350000 visiteurs chaque année. La prison de sécurité arrive ainsi après le Cap de Bonne espérance ou la Montagne de la Table en terme de fréquentation dans l'aire métropolitaine de CapeTown (D'angelo, 2014). Selon les enquêtes de Khumalo, Sebatlelo et Van der Merwe (2014), environ respectivement 67000 et 137000 visiteurs ont, eux, accédé aux sites du Constitution Hill et du Hector Pieterson Memorial \& Museum en 2012.

8. Le 24 septembre est notamment le jour de l'Heritage day.

9. A noter que Sharpely (2009), cite pour sa part l'exemple de l'incendie sur le bateau SS Morro Castle en 1934, par la suite échoué au large du New Jersey, et les nombreuses excursions festives qui ont suivi, comme premier exemple moderne de Dark Tourism. Notons qu'en Afrique du Sud, quelques sites relevant de nos jours du Dark tourism opéraient déjà sous l'apartheid : le musée Bo Kaap, les premiers Soweto tours ou les battlefields notamment...

10. Le fondateur de la société Disaster Tourism argumente en ce sens : «Les voyeurs sont ceux qui reçoivent l'information passivement et regardent les catastrophes à la télévision comme un spectacle ".

11. Comment peut-on être sûr que des sites comme le Taj Mahal ou les Catacombes aient été visités pour leur valeur historique plutôt que pour l'aspect mausolée ou sépulture qui leur est attaché (à moins d'aller sonder finement chaque personne arpentant ces lieux) ? En se faisant un brin provocateur, on pourrait arguer qu'un site comme l'Acropole à Athènes est aussi bien associé à la naissance de la démocratie qu'à la servitude qui y avait cours. Que faut-il retenir des tours Antebellum dans le sud de l'Amérique : une culture et des modes de vie typiques (ceux des plantations), ou les quartiers adjacents réservés aux esclaves?

12. Par exemple, Auschwitz s'est lancé dans un programme de modernisation mettant l'accent sur le destin individuel, l'héroïsme des victimes et la solidarité entre les prisonniers. À Belfast où le slogan "Come to Belfast and see our magnificent city » accueille le visiteur, une marche dans les rues à la découverte des murs de séparation, liés au conflit entre catholiques et protestants, nationalistes et unionistes, s'accompagne d'un message de paix et d'espoir. Il s'agit avant tout de positiver par son issue heureuse une histoire douloureuse, quitte parfois à sublimer le discours (retour à la normale ou « happy ending »).

\section{RÉSUMÉS}

Le champ de recherche relatif à la visite d'endroits liés aux épisodes dramatiques, à la souffrance et à la mort est relativement récent dans la sphère francophone. Il est davantage étudié dans le 
milieu anglophone, où le terme de Dark tourism («tourisme sombre ou noir ») a fini par faire autorité. Toutefois, un regard rarement distancié le concerne. Ce type d'attractions se veut aisément critiqué et critiquable. La controverse se cristallise généralement autour de la question suivante : le mariage entre la peine et l'histoire douloureuse et l'industrie du tourisme et des loisirs est-il concevable? Nous soutenons que la pluralité des enjeux sous-jacents doit empêcher tout débat tronqué et lesté d'idées reçues. Des ressorts multiples président à la volonté d'accéder aux prestations du Dark tourism. Nous nous proposons ici d'aborder quelques pistes de réflexions sur ce domaine de recherche, en nous appuyant sur des retours d'expériences du terrain sudafricain.

Research into visits associated with dramatic episodes, suffering and death is relatively recent among francophone scholars. But this theme has attracted more attention among English speakers where the term 'dark tourism' is used. Such attractions are easily open to criticism. Controversy usually crystallizes around whether a marriage between pain and suffering, and the tourism and recreation industry is really feasible. We maintain that the plurality of underlying challenges should prevent any simplistic debate; there are many issues involved in the recognition of 'dark tourism'. We advance some thoughts in this research domain, drawing on experiences in South Africa.

\section{INDEX}

Mots-clés : Dark tourism, Afrique du Sud, Johannesburg, expérience touristique, histoire, mémoire, patrimoine, motivations, enjeux

\section{AUTEUR}

\section{FABRICE FOLIO}

Fabrice Folio, Fabrice-jm.folio@univ-reunion.fr, est maître de conférences au Département de géographie, à l'Université de La Réunion et membre du CREGUR (Centre de Recherches et d'Etudes en Géographie de l'Université de La Réunion) - OIES (Océan Indien Espaces et Sociétés). 